\title{
Distributionally Robust Chance-Constrained Generation Expansion Planning
}

\author{
Pourahmadi, Farzaneh; Kazempour, Jalal; Ordoudis, Christos; Pinson, Pierre; Hosseini, Seyed Hamid
}

Published in:

IEEE Transactions on Power Systems

Link to article, DOI:

10.1109/TPWRS.2019.2958850

Publication date:

2020

Document Version

Peer reviewed version

Link back to DTU Orbit

Citation (APA):

Pourahmadi, F., Kazempour, J., Ordoudis, C., Pinson, P., \& Hosseini, S. H. (2020). Distributionally Robust Chance-Constrained Generation Expansion Planning. IEEE Transactions on Power Systems, 35(4), 2888 2903. https://doi.org/10.1109/TPWRS.2019.2958850

\section{General rights}

Copyright and moral rights for the publications made accessible in the public portal are retained by the authors and/or other copyright owners and it is a condition of accessing publications that users recognise and abide by the legal requirements associated with these rights.

- Users may download and print one copy of any publication from the public portal for the purpose of private study or research.

- You may not further distribute the material or use it for any profit-making activity or commercial gain

- You may freely distribute the URL identifying the publication in the public portal 


\title{
Distributionally Robust Chance-Constrained Generation Expansion Planning
}

\author{
Farzaneh Pourahmadi, Student Member, IEEE, Jalal Kazempour, Senior Member, IEEE, \\ Christos Ordoudis, Member, IEEE, Pierre Pinson, Fellow, IEEE, Seyed Hamid Hosseini, Member, IEEE
}

\begin{abstract}
This paper addresses a centralized generation expansion planning problem, accounting for both long- and shortterm uncertainties. The long-term uncertainty (demand growth) is modeled via a set of scenarios, while the short-term uncertainty (wind power generation) is described by a family of probability distributions with the same first- and second-order moments obtained from historical data. The resulting model is a distributionally robust chance-constrained optimization problem, which selects the conventional generating units to be built among predefined discrete options. This model includes a detailed representation of unit commitment constraints. To achieve computational tractability, we use a tight relaxation approach to convexify unit commitment constraints and solve the model with linear decision rules, resulting in a mixed-integer second-order cone program. It is observed that the proposed model exhibits better out-of-sample performance in terms of total expected system cost and its standard deviation compared to a chanceconstrained model that assumes a Gaussian distribution of shortterm uncertainty. A similar observation is made when comparing the proposed model against a chance-constrained program that uses empirical renewable power generation data with unknown type of distribution, recasting as either a robust optimization or a stochastic program.
\end{abstract}

Index Terms-Distributionally robust chance-constrained optimization, conic programming, linear decision rules, generation expansion planning, out-of-sample analysis, unit commitment.

\section{INTRODUCTION}

The development of capacity expansion planning models is a complex task in power systems with high penetration of weather-dependent renewable energy sources. The first reason for such a complexity is that the planning models should accurately characterize the renewable power generation uncertainty throughout the long-term planning horizon. The second reason is that the planning models should take into account the operational constraints of power systems as accurate as possible, since these constraints are more often binding with renewable power generation variability. In this paper, we focus on planning problems in terms of generation assets, and develop a generation expansion planning (GEP) model accounting for the aforementioned complexities.

In a market context, the generation investment falls into the internal decisions of power producers, who in general seek to maximize their own profit [1]. However, a centralized GEP model with a single decision-maker, which is the focus of

F. Pourahmadi and S. H. Hosseini are with the Department of Electrical Engineering, Sharif University of Technology, Tehran 11365-11155, Iran (emails: Pourahmadi_f@ee.sharif.edu; hosseini@sharif.edu).

J. Kazempour, $\bar{C}$. Ordoudis, and P. Pinson are with the Department of Electrical Engineering, Technical University of Denmark, Kgs. Lyngby 2800, Denmark (e-mails: seykaz@elektro.dtu.dk; christos.ordoudis@gmail.com; ppin@elektro.dtu.dk). this paper, can be still useful for market studies. The reason for this is that the centralized model can serve as an ideal benchmark, and provide the market regulators with insights into the optimal planning decisions for the whole system. The market regulators may then design proper policies ${ }^{1}$ to incentivize power producers to make generation investment decisions, which are optimal from the social welfare perspective.

The sources of uncertainty in expansion planning studies can be generally categorized into long- and short-term uncertainties [1], [3]. The former corresponds to uncertain sources to be realized in the long run, e.g., load growth, future share of renewables in power systems, future fuel cost, and policy regulations. In contrast, the short-term uncertainties pertain to uncertain sources to be realized in the operational stage, e.g., power generation of renewables and consumption of loads. In the existing literature, scenario-based stochastic programming and robust optimization are the two techniques that are commonly used to characterize uncertainties in GEP models [1], but each of those techniques has its own shortcomings [4]-[6]. The performance of stochastic GEP models highly depends on the set of scenarios representing the sources of uncertainty. It is crucial to ensure that the set of scenarios describes well the true probability distribution of the uncertainty [7]. This usually requires to embed a very large number of scenarios in the model, which may result in computational intractability. A reduction in the number of scenarios restores the tractability, but at the potential cost of naive representation of the uncertainty in stochastic GEP models, resulting in a weak out-of-sample performance [8]. On the other hand, the GEP models based on robust optimization make the optimal planning decisions against the worst-case realization of a prescribed uncertainty set, which may achieve an over-conservative solution. It is also complicated to define a proper uncertainty set, accounting for all potential distributions of uncertainties.

In addition to aforementioned challenges in characterizing uncertainty, there is another challenge caused by two simplifications that are commonly made in long-term planning studies, which might be no longer valid. The first common simplification is to ignore chronological variations of the netload, i.e., demand minus renewable power generation, by using a net-load duration curve over the planning horizon [9]. The second one is to constrain the GEP optimization model by economic dispatch limits only, discarding the operational unit commitment (UC) constraints, e.g., start-up, shut-down,

\footnotetext{
${ }^{1}$ An example of such policies is to design capacity market or risk trading mechanisms [2].
} 
and minimum up/down time limits. There are several recent studies showing that these simplifications are distorting the expansion planning decisions in power systems with high renewable power penetration [5], [10]-[14]. In particular, these simplifications may lead to an underestimation of the need for new generation capacity, and eventually a significant increase in the actual operational cost of the system [14].

Based on all these challenges, we address the following three research questions: How can the renewable generation uncertainty be properly characterized in a GEP model while its true probability distribution is unknown? How to ensure that the operational limits including UC constraints are properly enforced in the GEP model while maintaining the computational tractability? And, how important is it to take into account the potential spatial and temporal correlations of renewable generation uncertainty in the GEP model?

To properly address all these questions, we propose a distributionally robust chance-constrained GEP model. In this model, an ambiguity set embodies a family of probability distributions, all with identical first- and second-order moments, i.e., mean and covariance, obtained from historical data $^{2}$. Each probability distribution in the ambiguity set represents a potential distribution of short-term uncertainties. In addition, the long-term uncertainties are modeled using a set of scenarios drawn from expert knowledge. The proposed model determines the optimal generation expansion plans, resulting in the minimum total system cost, i.e., the sum of planning and operational costs of the system. This cost is minimum in expectation ${ }^{3}$, accounting for the set of long-term scenarios and the worst distribution of short-term uncertainties. This worst distribution within the ambiguity set is endogenously determined by the proposed model. Our proposed GEP model is constrained by expansion limits as well as operational limits. We use a tight convex relaxation of $\mathrm{UC}$ constraints that improves the computational tractability by relaxing the discrete nature of UC variables, e.g., on/off and start-up status of conventional generating units. We enforce the operational limits, except for nodal power balance conditions, in the form of distributionally robust chance constraints. This enables adjusting the conservativeness of planning decisions against the

\footnotetext{
${ }^{2}$ In the existing literature of distributionally robust optimization, two distinct types of ambiguity set are extensively used: moment-based and metric-based In the former, the ambiguity set includes all probability distributions, whose moments, e.g., mean and covariance, are identical or close enough to those of empirical data [15]-[17]. In the latter, the ambiguity set is defined as a ball in the space of distributions, such that the empirical distribution is considered in the center, and the ball is constructed around the center using a probability distance function, such as $\phi$-divergence, Kullback-Leibler divergence, or Wasserstein metric [18], [19]. Although the metric-based ambiguity set guarantees a comparatively stronger out-of-sample performance, the moment-based one provides better tractability features [19]. Besides, a distributionally robust optimization with moment-based ambiguity set is in general more tractable in comparison to its stochastic counterpart, whereas a distributionally robust model build upon Wasserstein metric is generally more computationally expensive [19]. A comprehensive review for distributionally robust optimization is available in [20]. In this paper, we limit our attention to distributionally robust optimization with a moment-based ambiguity set, where the values of the first two moments are exactly known.

${ }^{3}$ This implies that the solution obtained from the proposed model does not necessarily result in the minimum system cost for each individual long-term scenario, or for a specific sample captured from the worst distribution of the short-term uncertainty, or in expectation under any other distribution in the ambiguity set.
}

worst distribution of short-term uncertainties. Adopting linear decisions rules [21] allows recasting the proposed model as a mixed-integer second-order cone program with good computational performance. Note that the resulting model is mixedinteger due to the discrete representation of expansion options. Otherwise, with a simpler but continuous representation of candidate units to be built, the resulting model would be a second-order cone program without integrality constraints.

There exist several works in the literature, e.g., [10]-[12], that present various types of approximation for UC constraints and account for the variability of net-load in the GEP model in a computationally tractable manner. However, the renewable power generation uncertainty is neglected in [10]-[12]. Some other studies characterize uncertainties in GEP models using either scenario-based stochastic programming, e.g., [4], [5], [14], [22], or robust optimization, e.g., [6], with or without enforcing UC constraints. To the best of our knowledge, there is no other work in the existing literature proposing a distributionally robust chance-constrained GEP model. References [23] and [24] use a distributionally robust optimization with a moment-based ambiguity set for expansion planning problems, but their purpose is to determine the optimal investment in transmission assets. It is worth mentioning that the ambiguity set in [23] and [24] considers the first moment information of uncertainties only. Therefore, these models miss the potential temporal and spatial correlations of uncertainties. In addition, they build a relatively large ambiguity set that may result in a very conservative solution, especially without enforcing chance constraints. Also, these two papers neglect UC constraints. All different technical aspects of aforementioned works are summarized and compared in Table I.

In addition to [23] and [24], distributionally robust optimization has been recently applied to an extensive range of applications in power systems, such as UC [25]-[27], optimal power flow [28]-[32], bidding strategy [33], hydroelectric reservoir optimization [34], congestion management [35], energy management in microgrids [36], and distribution system planning [37].

Given the context above, the main contributions of this paper are as follows: we develop a moment-based distributionally robust GEP model, while leveraging a tight relaxation of UC constraints. The distributionally robust chance constraints are incorporated into the proposed GEP model within the operational stage. The proposed model accounts for both long- and short-term uncertainties as well as flexibility requirements, and adjusts the conservativeness of the solution by varying the desired confidence level of chance constraints. By considering an ambiguity set including the second-order moment information obtained from empirical data, the existing spatial and temporal correlations of short-term uncertainties are properly modeled. Our findings show that the proposed GEP model based on a distributionally robust chance-constrained optimization exhibits a better out-of-sample performance compared to a similar model relying on a chance-constrained optimization following a Gaussian distribution. The same finding still holds when we consider a chance-constrained optimization using the empirical distribution, which is recast as either a robust optimization or a stochastic program.

The remainder of this paper is structured as follows. Section 
TABLE I

The Comparison of Relevant Works Proposed in The Literature and The Model Proposed in This Paper

\begin{tabular}{|c|c|c|c|c|c|c|}
\hline Ref. & Problem & $\begin{array}{l}\text { Operational } \\
\text { constraints }\end{array}$ & $\begin{array}{l}\text { Uncertainty modeling } \\
\text { technique }\end{array}$ & $\begin{array}{l}\text { Correlation of } \\
\text { uncertainties }\end{array}$ & $\begin{array}{c}\text { Out-of-sample } \\
\text { analysis }\end{array}$ & $\begin{array}{l}\text { Resulting } \\
\text { model }\end{array}$ \\
\hline$[10]-[12]$ & $\mathrm{GEP}^{4}$ & Unit commitment & Deterministic & No & No & MILP $^{5}$ \\
\hline$[4],[22]$ & GEP & Economic dispatch & Stochastic programming & No & No & MILP \\
\hline [5], [14] & GEP & Unit commitment & Stochastic programming & No & No & MILP \\
\hline [6] & GEP & Economic dispatch & Robust optimization & No & Yes & MILP \\
\hline [23], [24] & TEP $^{6}$ & Economic dispatch & Distributionally robust optimization & No & Yes & MILP \\
\hline This paper & GEP & Unit commitment & Distributionally robust chance-constrained optimization & Yes & Yes & $\mathrm{MISOCP}^{7}$ \\
\hline
\end{tabular}

II first provides preliminaries on expansion time horizon and uncertainty modeling, and then lists our assumptions. Section III presents the proposed distributionally robust chanceconstrained GEP model. Section IV explains the solution methodology. Section V provides a comprehensive case study based on the IEEE 118-node test system. Section VI concludes the paper. Appendix A presents a nomenclature. Additional five appendices provide technical information that will be required throughout the paper.

\section{Preliminaries And Assumptions}

\section{A. Expansion Time Horizon}

Two distinct approaches are commonly used in expansion planning studies to model the planning time horizon: static (one-year) and dynamic (multi-year) [1]. In the static expansion approach, a single target year only, e.g., the 20th year from now, is considered as the expansion time horizon. This model determines the optimal generation assets required to be built by the target year, but does not find the optimal years of expansion from now to the target year. On the contrary, the dynamic expansion approach divides the planning time horizon into multiple years, and determines the optimal time of expansion plans in addition to new capacities to be built. Further information about these two approaches are available in [1]. The proposed GEP model in this paper takes into account a static expansion approach with a single target year. This means that we simply consider annualized expansion and operational costs. It is straightforward to extend the proposed model using a dynamic approach, but the computational time may drastically increase and a decomposition method will be eventually required.

It is common in expansion planning studies to consider a load duration curve (LDC), or a net-load duration curve, as an approximation of hourly load curve during the expansion time horizon [38]-[40]. The aim of such LDC-based expansion models is to meet the capacity adequacy requirements only. However, the main drawback is that it ignores the chronological sequence of net-load profile, and therefore the operational flexibility limitations cannot be modeled. To resolve this drawback while maintaining the computational tractability, the net-load during the planning horizon can be represented through a set of representative operating periods (days or weeks) [41]-[43]. In this paper, we use a set of representative days $r \in R$, each including 24 hours. We assign a weight to each representative day $r$, indicating the number of days in the target year, whose net-load profile is represented by day $r$.

\section{B. Uncertainty Modeling}

In general, there is a lack of information related to the probability distribution of long-term uncertainties, as discussed in [24]. Therefore, we model the uncertainty of demand growth and future capacity of renewables through a finite set of possible scenarios $s \in S$ drawn by expert knowledge.

For each long-term scenario $s$, representative day $r \in R$ and hour $t \in\{1,2, \ldots, 24\}$, we model the short-term net-load uncertainty by means of an ambiguity set, i.e., a family of possible probability distributions of the net-load uncertainty, which is built upon the information associated with the firstand second-order moments, i.e., mean and covariance, of available historical data. We assume that the exact values for first- and second-order moments can be estimated by available historical data, and leave modeling of inexact moments to future work ${ }^{8}$.

In the rest of the paper, we use upper-case bold letters for matrices and lower-case bold letters for vectors. In addition, $(.)^{\top}$ represents the transpose operator, whereas $\mathbb{R}$ shows the set of real numbers, and $\mathbb{R}_{+}$refers to the set of non-negative real numbers.

We consider $Z$ number of short-term uncertain sources. The power generation of uncertain sources in the short run is modeled by $\mathbf{m}_{s r t}+\gamma_{s r t}$, where $\mathbf{m}_{s r t} \in \mathbb{R}_{+}^{Z}$ is the mean of power generation forecast of uncertain sources. The forecast error $\gamma_{s r t} \in \mathbb{R}^{Z}$ under a given probability distribution is a random variable with the mean vector $\boldsymbol{\mu}_{\text {srt }} \in \mathbb{R}^{Z}$ and the covariance matrix $\boldsymbol{\Sigma}_{\text {srt }} \in \mathbb{R}^{Z \times Z}$. The ambiguity set $\mathcal{P}_{\text {srt }}$ associated with long-term scenario $s$, representative day $r$, and hour $t$ writes as

$\mathcal{P}_{\text {srt }}=\left\{D_{\text {srt }} \in \Psi_{\text {srt }}\left(\mathbb{R}^{Z}\right): \mathbb{E}^{D}(\boldsymbol{\gamma})=\boldsymbol{\mu}_{\text {srt }}, \mathbb{E}^{D}\left(\boldsymbol{\gamma}^{\top} \boldsymbol{\gamma}\right)=\boldsymbol{\Sigma}_{\text {srt }}\right\}$,

where $D_{s r t}$ is a probability distribution, belonging to family of distributions $\Psi_{s r t}\left(\mathbb{R}^{Z}\right)$, all with the same first- and secondorder moments, including the uncertainty information for all

\footnotetext{
${ }^{8}$ Note that inexact moments may complicate the model, and eventually yield a semi-definite program [44]. It might also be of interest to incorporate more information into the ambiguity set, e.g., extra moments, a support set including physical bounds for wind power generation, or unimodality and logconcavity properties [45]. This additional information excludes the potentially non-realistic distributions from the ambiguity set and increases the quality of results. However, it may again complicate the model, and therefore we leave it for future extensions.
} 
short-term uncertain sources. Note that $\mathbb{E}^{D}[$.$] is the expec-$ tation operator wherein the uncertain parameter $\gamma_{\text {srt }}$ follows distribution $D_{s r t}$. For notational convenience, the indices of $\gamma_{s r t}$ are dropped in (1) and in the rest of the paper. From now on, we also drop indices of $D_{\text {srt }}$, and assume that the mean vector of forecast error is zero, i.e., $\boldsymbol{\mu}_{s r t}=0, \forall s, r, t$.

\section{Additional Assumptions}

Similar to the majority of expansion planning studies in the literature, we use a linearized loss-less DC approximation of power flow across the network. We assume that the topology of the power network is unchanged throughout the planning horizon. We also discard quick-start units, meaning that the on/off commitment status of all conventional generating units are determined before the short-term uncertainty realization, and this status cannot be changed in real time. However, these commitment variables are dependent on long-term scenarios. Finally, we assume that each renewable energy source is scheduled at its mean power output with a zero operational cost.

\section{PROPOSED MODEL}

In this section, we present the proposed distributionally robust chance-constrained (DRCC) model for the GEP problem. We first define all notation. A list of notation is also provided in Appendix A.

\section{A. Notation}

We consider $G$ number of existing and candidate conventional generating units, and use index $i \in\{1,2, \ldots, G\}$ for these units. Note that $G^{C}$ out of $G$ units are candidate units to be built. For each unit $i$, parameters $\bar{p}_{i} \in \mathbb{R}_{+}$and $\underline{p}_{i} \in \mathbb{R}_{+}$indicate its maximum and minimum production level, respectively. Parameters $\bar{r}_{i} \in \mathbb{R}_{+}$and $\underline{r}_{i} \in \mathbb{R}_{+}$represent the maximum ramp-up and ramp-down capability of unit $i$, respectively. Parameter $r_{i} \in \mathbb{R}_{+}$indicates the ramp rate limit of unit $i$ in start-up and shut-down hours only. Parameter vectors $\mathbf{c} \in \mathbb{R}_{+}^{G}$ and $\mathbf{h} \in \mathbb{R}_{+}^{G}$ refer to the production cost and the start-up cost of generating units, respectively. Parameter vectors $\underline{\mathbf{v}} \in \mathbb{R}_{+}^{G}$ and $\overline{\mathbf{v}} \in \mathbb{R}_{+}^{G}$ give the minimum up- and down-time of generating units, respectively. We also consider $B$ demands. For given demand growth scenario $s$ to be realized in the long run, we assume that the short-term load $\mathbf{d}_{s r t} \in \mathbb{R}_{+}^{B}$ is inelastic to price and exactly known. With this assumption, the netload uncertainty in the short run boils down to renewable power generation uncertainty. Parameter vector $\mathbf{k} \in \mathbb{R}_{+}^{G^{C}}$ indicates the annualized capital cost of candidate units. $\mathrm{Pa}$ rameter $\bar{f}_{l} \in \mathbb{R}_{+}$refers to the capacity of transmission line $l$. The power network consists of $U$ nodes and $L$ transmission lines. The power flow throughout the network is determined using a power transfer distribution factor matrix $\mathbf{H} \in \mathbb{R}^{L \times U}$, which defines the power flow as a linear function of nodal injections and withdrawals. More specifically, we define three matrices $\mathbf{H}^{\mathrm{G}} \in \mathbb{R}^{L \times G}, \mathbf{H}^{\mathrm{W}} \in \mathbb{R}^{L \times Z}$, and $\mathbf{H}^{\mathrm{D}} \in \mathbb{R}^{L \times B}$ that incorporate the mapping of conventional generating units, renewable energy sources, and demands into the network, respectively. Besides, $\mathbf{1}$ and $\mathbf{0}$ are the vector of ones and zeros, respectively.
We now define the decision variables. Generation expansion decisions are binary variables, meaning that the optimal decisions are selected among predefined expansion options. Binary variable vector $\mathbf{y} \in\{0,1\}^{G^{C}}$ determines which candidate units should be built. As operational stage variables, variable vector $\mathbf{x}_{\text {srt }}=\left[x_{1 s r t}, \ldots, x_{i s r t}, \ldots, x_{G s r t}\right]^{\top} \in\{0,1\}^{G}$ indicates the on/off commitment status of generating units. Variable vector $\mathbf{u}_{\text {srt }}=\left[u_{1 s r t}, \ldots, u_{\text {isrt }}, \ldots, u_{G s r t}\right]^{\top} \in\{0,1\}^{G}$ gives the start-up status of units. Finally, variable vector $\mathbf{p}_{s r t}(\boldsymbol{\gamma})=\left[p_{1 s r t}(\gamma), \ldots, p_{\text {isrt }}(\gamma), \ldots, p_{\text {Gsrt }}(\gamma)\right]^{\top} \in \mathbb{R}_{+}^{G}$ represents the production level of units. Note that the commitment and start-up variable vectors $\mathbf{x}_{\text {srt }}$ and $\mathbf{u}_{\text {srt }}$ are dependent on long-term uncertainty, but are independent of short-term uncertainty, i.e., these decisions are made before the time that the short-term uncertainty is realized. However, production variable vector $\mathbf{p}_{\text {srt }}(\gamma)$ is dependent on both long- and shortterm uncertainties. Therefore, conventional units can alter their production level in real-time to contribute to coping with power balance deviations caused by renewable energy sources.

\section{B. Formulation}

The proposed DRCC model includes (2) to (5). The objective function seeks to minimize the total system cost, which is the expansion cost plus the worst expected operational cost, i.e.,

$$
\min _{\mathbf{y}} \mathbf{k}^{\top} \mathbf{y}+\sum_{s r t} \pi_{s} \max _{D \in \mathcal{P}_{s r t}} \kappa_{r} Q_{s}(\mathbf{y}),
$$

where the first term, i.e., $\mathbf{k}^{\top} \mathbf{y}$, is the annualized expansion cost, whereas the second term is the annualized expected operational cost under the worst probability distribution $D \in \mathcal{P}_{\text {srt }}$. Note that $\pi_{s} \in \mathbb{R}_{+}$is the probability of long-term scenario $s$, whereas $\kappa_{r} \in \mathbb{R}_{+}$is the weight of representative day $r$. Finally, $Q_{s}(\mathbf{y})$ represents the optimal annualized expected operational cost under probability distribution $D$ for given investment decision $\mathbf{y}$ and long-term scenario $s$. This cost includes production and start-up costs of conventional units, and writes as

$$
Q_{s}(\mathbf{y})=\min _{\mathbf{p}, \mathbf{x}, \mathbf{u}} \mathbb{E}^{D}\left[\mathbf{c}^{\top} \mathbf{p}_{s r t}(\gamma)+\mathbf{h}^{\top} \mathbf{u}_{s r t}\right] .
$$

Recall that $\mathbb{E}^{D}[$.$] is the expectation operator. Note that the$ objective function is indeed a three-stage min-max-min problem, which will be recast as a single-stage problem by using linear decision rules - it will be explained later in Section IV and Appendix B. The first set of constraints is

$$
\begin{aligned}
& x_{i s r t} \leqslant \mathbf{y}_{i}, \quad \forall i \in G^{C}, s, r, t \\
& -\mathbf{x}_{s r(t-1)}+\mathbf{x}_{s r t}-\mathbf{x}_{s r \tau} \leqslant \mathbf{0}, \\
& \forall \tau \in\{t, \ldots, \overline{\mathbf{v}}+t-1\}, \forall s, r, t \\
& \mathbf{x}_{s r(t-1)}-\mathbf{x}_{s r t}+\mathbf{x}_{s r \tau} \leqslant \mathbf{1}, \\
& \forall \tau \in\{t, \ldots, \mathbf{v}+t-1\}, \forall s, r, t \\
& -\mathbf{x}_{s r(t-1)}+\mathbf{x}_{s r t}-\mathbf{u}_{s r t} \leqslant \mathbf{0}, \quad \forall s, r, t \\
& \mathbf{1}^{\top} \mathbf{p}_{s r t}(\gamma)+\mathbf{1}^{\top}\left(\mathbf{m}_{s r t}+\gamma\right)=\mathbf{1}^{\top} \mathbf{d}_{s r t}, \mathbb{P}-\text { a.s. } \forall s, r, t \\
& \mathbf{y} \in\{0,1\} \\
& \mathbf{x}_{s r t}, \mathbf{u}_{s r t} \in\{0,1\}, \quad \forall s, r, t .
\end{aligned}
$$

Constraint (4a) enforces the commitment status of candidate unit $i \in G^{C}$ to be zero if it is not selected to be built. 
Constraints (4b) and (4c) impose the minimum up- and downtime limits of units, whereas (4d) represents the units' state transition. Constraint (4e) enforces the power balance almost surely with probability 1 for any realization of uncertainty [21]. Although this constraint is dependent on short-term uncertainty $\gamma$, it is treated as the most important operational constraint of the problem, meaning that it should be respected under any potential realization. Finally, (4f) and (4g) declare the integrality conditions.

In addition to (4), the proposed problem is constrained by a set of distributionally robust chance constraints. Unlike the operational constraint (4e), capacity and ramping limits of generating units as well as capacity limits of transmission lines are imposed through individual distributionally robust chance constraints. This allows adjusting the conservativeness of the proposed model. For example, the capacity constraint of unit $i$ is enforced by

$$
\min _{D \in \mathcal{P}_{\text {srt }}} \mathbb{P}\left[p_{\text {isrt }}(\gamma) \leqslant \bar{p}_{i} x_{i s r t}\right] \geqslant 1-\epsilon_{i}, \quad \forall i, s, r, t,
$$

where $\mathbb{P}[$.$] is the probability operator wherein the uncertain$ parameter $\gamma$ follows the worst distribution $D$ within ambiguity set $\mathcal{P}_{\text {srt }}$. This specific distributionally robust chance constraint implies that under the worst distribution in ambiguity set $\mathcal{P}_{\text {srt }}$, the probability of meeting the capacity constraint should be greater than or equal to $1-\epsilon_{i}$, where predefined parameter $\epsilon_{i} \in \mathbb{R}_{+}$lies within zero and one. It is worth mentioning that the worst distribution $D$ in (5a) is not necessarily identical to that of objective function (2) or that of any other distributionally robust chance constraint. Similarly, the rest of individual distributionally robust chance constraints write as

$$
\begin{aligned}
& \min _{D \in \mathcal{P}_{s r t}} \mathbb{P}\left[p_{i s r t}(\gamma) \geqslant \underline{p}_{i} x_{i s r t}\right] \geqslant 1-\epsilon_{i}, \quad \forall i, s, r, t \\
& \min _{D \in \mathcal{P}_{s r t}} \mathbb{P}\left[p_{i s r t}(\gamma)-p_{i s r(t-1)}(\gamma) \leqslant \bar{r}_{i} x_{i s r(t-1)}\right. \\
& \quad+r_{i}\left(1-x_{i s r(t-1)}\right) \geqslant 1-\epsilon_{i}, \quad \forall i, s, r, t \\
& \min _{D \in \mathcal{P}_{s r t}} \mathbb{P}\left[p_{i s r(t-1)}(\gamma)-p_{i s r t}(\gamma) \leqslant \underline{r}_{i} x_{i s r t}\right. \\
& \left.\quad+r_{i}\left(1-x_{i s r t}\right)\right] \geqslant 1-\epsilon_{i}, \quad \forall i, s, r, t \\
& \min _{D \in \mathcal{P}_{s r t}} \mathbb{P}\left[\mathbf{H}_{l}^{\mathrm{G}} \mathbf{p}_{s r t}(\gamma)+\mathbf{H}_{l}^{\mathrm{W}}\left(\mathbf{m}_{s r t}+\gamma\right)\right. \\
& \left.\quad-\mathbf{H}_{l}^{\mathrm{D}} \mathbf{d}_{s r t} \leqslant \bar{f}_{l}\right] \geqslant 1-\epsilon_{l}, \quad \forall l, s, r, t \\
& \min _{D \in \mathcal{P}_{s r t}} \mathbb{P}\left[\mathbf{H}_{l}^{\mathrm{G}} \mathbf{p}_{s r t}(\gamma)+\mathbf{H}_{l}^{\mathrm{W}}\left(\mathbf{m}_{s r t}+\gamma\right)\right. \\
& \left.\quad-\mathbf{H}_{l}^{\mathrm{D}} \mathbf{d}_{s r t} \geqslant-\bar{f}_{l}\right] \geqslant 1-\epsilon_{l}, \quad \forall l, s, r, t .
\end{aligned}
$$

The distributionally robust chance constraint $(5 b)$ imposes the minimum production level of units, whereas (5c) and (5d) restrict the ramping of units. Finally, (5e) and (5f) enforce the capacity of transmission lines. Note that the subscript in $\mathbf{H}_{l}^{(.)}$ picks a row corresponding to transmission line $l$ from matrix $\mathbf{H}^{(.)}$. The next section explains how to solve the proposed DRCC problem (2)-(5).

\section{Solution Methodology}

The proposed DRCC model (2)-(5) is computationally hard to solve or even intractable due to the infinite-dimensional nature of the problem. The mixed-integer nature of the problem makes the problem hard to solve too, especially if the number of binary variables is relatively high. For achieving tractability, we solve the proposed model in linear decision rules [21], and use a tight relaxation approach to convexify UC constraints [46]. We first introduce an approximation approach to convexify UC constraints. We then develop an affine policy for conventional units, built upon the theory of linear decision rules, to analytically reformulate the distributionally robust chance constraints.

\section{A. Tight Relaxation of Unit Commitment Constraints}

Expansion decisions $\mathbf{y}$ are the most important variables of the proposed model, and therefore their integrality conditions need to be preserved. On the contrary, it is computationally appealing to relax the integrality conditions of UC constraints in the operational stage, but as tight as possible. The binary variables of UC constraints are on/off commitment status $\mathbf{x}_{\text {srt }}$ and start-up status $\mathbf{u}_{\text {srt }}$ of generating units. To enhance the computational tractability, we use a tight convex relaxation approach, similar to the one proposed in [12] and [46], which substitutes the feasible set of each generating unit by a tractable approximation of its convex hull ${ }^{9}$. In this approach, we first relax each operational-stage binary variable to lie within zero and one, i.e.,

$$
\mathbf{0} \leqslant \mathbf{x}_{s r t} \leqslant \mathbf{1} ; \quad \mathbf{0} \leqslant \mathbf{u}_{s r t} \leqslant \mathbf{1}, \quad \forall s, r, t .
$$

Then, additional inequalities are embedded to tighten the relaxation [48]. Similar to capacity and ramping constraints of generating units in (5), we consider these additional tightening constraints in form of distributionally robust chance constraints:

$$
\begin{aligned}
& \min _{D \in \mathcal{P}_{s r t}} \mathbb{P}\left[p_{i s r(t-1)}(\gamma) \leqslant r_{i} x_{i s r(t-1)}\right. \\
& \left.\left.\quad+\left(\bar{p}_{i}-r_{i}\right)\left(x_{i s r t}-u_{i s r t}\right)\right)\right] \geqslant 1-\epsilon_{i}, \forall i, s, r, t \\
& \min _{D \in \mathcal{P}_{s r t}} \mathbb{P}\left[p_{i s r t}(\gamma) \leqslant \bar{p}_{i} x_{i s r t}\right. \\
& \left.\left.\quad-\left(\bar{p}_{i}-r_{i}\right) u_{i s r t}\right)\right] \geqslant 1-\epsilon_{i}, \quad \forall i, s, r, t \\
& \min _{D \in \mathcal{P}_{s r t}} \mathbb{P}\left[p_{i s r t}(\gamma)-p_{i s r(t-1)}(\gamma) \leqslant\left(\underline{p}_{i}+\bar{r}_{i}\right) x_{i s r t}\right. \\
& \left.\quad-\underline{p}_{i} x_{i s r(t-1)}-\left(\underline{p}_{i}+\bar{r}_{i}-r_{i}\right) u_{i s r t}\right] \geqslant 1-\epsilon_{i}, \forall i, s, r, t \\
& \min _{D \in \mathcal{P}_{s r t}} \mathbb{P}\left[p_{i s r t}(\gamma)-p_{i s r(t-1)}(\gamma) \geqslant\left(\underline{p}_{i}+\underline{r}_{i}-r_{i}\right) u_{i s r t}\right. \\
& \left.\quad-r_{i} x_{i s r(t-1)}+\left(r_{i}-\underline{r}_{i}\right) x_{i s r t}\right] \geqslant 1-\epsilon_{i}, \forall i, s, r, t .
\end{aligned}
$$

As a result, the proposed formulation for GEP can now be stated as the collection of (2)-(3), (4a)-(4f), (5)-(7).

\section{B. Linear Decision Rules}

To further mitigate the complexity of the proposed model, the recourse actions of conventional units are approximated with linear decision rules ${ }^{10}$ [21]. In this way, the production of each conventional unit $i$, i.e., $p_{i s r t}(\gamma)$ is described by $p_{\text {isrt }}+\left(\mathbf{1}^{\top} \gamma\right) \alpha_{i s r t}$. Note that $p_{\text {isrt }} \in \mathbb{R}_{+}$is the tentative day-ahead schedule of unit $i$, whereas the recourse action

\footnotetext{
${ }^{9} \mathrm{We}$ do not investigate the impacts of this relaxation on pricing, but refer interested readers to [47].

${ }^{10}$ This approximation can be improved by using generalized decision rules [49], but at the cost of increased complexity. This extension is left to future work.
} 
$\left(\mathbf{1}^{\top} \gamma\right) \alpha_{\text {isrt }}$ represents the linear response of unit $i$ in real-time to the uncertain renewable power generation. In particular, we refer to variable $\alpha_{i s r t} \in \mathbb{R}$ as the participation factor of unit $i$ towards mitigation of the unplanned power balance deviation in real-time due to the total forecast error of renewable energy sources, i.e., $\mathbf{1}^{\top} \gamma$. The optimal value of $\alpha_{i s r t}$ for each unit lies within -1 and 1 . Using this affine control policy for conventional units while assuming $\boldsymbol{\mu}_{s r t}=0, \forall s, r, t$, we reformulate objective function (2), power balance constraint (4e) and distributionally robust chance constraints (5) and (7) to make the original problem tractable. The details of this reformulation are given in Appendix B.

It is worth noting that ramping chance constraints $(5 c)-(5 d)$ and $(7 c)-(7 d)$ impose inter-temporal coupling between dispatch decisions. For these constraints, the uncertainty parameter vector contains both vectors $\gamma_{s r t}$ and $\gamma_{s r(t-1)}$ such that

$$
\hat{\gamma}_{s r t}=\left[\begin{array}{c}
\gamma_{s r t} \\
\gamma_{s r(t-1)}
\end{array}\right]
$$

where $\hat{\gamma}_{\text {srt }} \in \mathbb{R}^{2 Z}$. As a result, another covariance matrix needs to be defined to model the effect of temporal correlation on ramping constraints that can be written as

$$
\widehat{\boldsymbol{\Sigma}}_{s r t}=\left[\begin{array}{cc}
\boldsymbol{\Sigma}_{s r t} & \boldsymbol{\Upsilon}_{s r(t, t-1)} \\
\boldsymbol{\Upsilon}_{s r(t, t-1)} & \boldsymbol{\Sigma}_{s r(t-1)}
\end{array}\right],
$$

where $\widehat{\boldsymbol{\Sigma}}_{\text {srt }} \in \mathbb{R}^{2 Z \times 2 Z}$ includes not only the spatial correlation of wind farms, but also their temporal correlation between hours $t$ and $t-1$.

The original DRCC problem boils down to a mixed-integer second-order cone program, whose formulation is given in Appendix C.

\section{Numerical STUdy}

We use a case study based on a modified version of the IEEE 118-node test system [50] including 19 existing conventional units, 2 existing wind farms, 99 demands, and 186 transmission lines. By existing, we refer to those conventional or wind power units that either exist now and will not be phased out by the target year, or units that do not exist now, but it is certain that they will be built by the target year. In addition to the existing conventional and wind units, we consider 22 candidate conventional units that can be built.

All simulations are run on an $\operatorname{Intel}(\mathrm{R}) \mathrm{Xeon}(\mathrm{R})$ E5-1650 with 12 processors clocking at $3.50 \mathrm{GHz}$ and $32 \mathrm{~GB}$ of RAM. The source code implemented in Matlab using YALMIP and solved by Gurobi 7.5.1 is available in the online companion [51]. Depending on the values assigned for $\epsilon$ in distributionally robust chance constraints, the CPU time varies from 2 to 3 hours. Further details about computational issues will be provided later in Table III.

\section{A. Input Data}

We consider several types of conventional units, including coal, nuclear, combined cycle gas turbine (CCGT) and gas turbine (GT). Their technical parameters are given in Table II. The total capacity of existing conventional units is 6,466 MW. Full details about technical characteristics of generating units,
TABLE II

\begin{tabular}{|c|c|c|c|c|c|}
\hline Type & Coal & Nuclear & CCGT & GT1 & GT2 \\
\hline Oper. cost (\$/MWh) & 18.72 & 10.33 & 19.32 & 38.47 & 48.47 \\
\hline Start-up cost $(\$ / \mathrm{MW})$ & 54.11 & 100 & 16.23 & 25.14 & 28.14 \\
\hline Exp. cost $\left(10^{3} \$ / \mathrm{MW}\right.$-year $)$ & 174.7 & 224 & 60.1 & 48.5 & 38.5 \\
\hline Min. generation (p.u.) ${ }^{\dagger}$ & 0.75 & 0.9 & 0.4 & 0.27 & 0.25 \\
\hline Ramp rate (p.u. $)^{\dagger}$ & 0.3 & 0.1 & 0.5 & 0.7 & 1 \\
\hline Min. up time (h) & 24 & 48 & 6 & 1 & 1 \\
\hline Min. down time $(\mathrm{h})$ & 12 & 24 & 12 & 1 & 1 \\
\hline Nr. of existing units & 4 & 2 & 4 & 2 & 7 \\
\hline Nr. of candidate units & 4 & 2 & 6 & 4 & 6 \\
\hline
\end{tabular}

DATA For CONVENTIONAL GENERATING Units

${ }^{\dagger}$ It is given as a percentage of unit's capacity.

transmission lines, load profile of demands, and wind power profile of two farms are given in the online companion [51].

Using a K-means clustering technique, we provide net-load data for 10 days, each with a different profile, to represent the target year. The reason for selecting 10 representative days is that with increasing the number of these days, the investment solution does not change significantly.

Without loss of generality, two long-term scenarios with the same probabilities are considered ${ }^{11}$. It is assumed that the load level of the target year in the first and second scenarios is 3.4 and 4 times of the existing load level with the same pattern, respectively. The capacity of two wind farms in the target year is assumed to be 2,400 MW and 3,000 MW under the first long-term scenario, while it is assumed to be 5,355 MW and 6,120 MW for the second scenario. Therefore, the wind penetration level, i.e., the yearly contribution of wind power to supply demand, is $35 \%$ under both long-term scenarios.

For each wind farm, we generate 10,000 power trajectories from the historical data, each containing the wind power profile of that farm over all hours of the 10 representative days. We then split them into two sets of 5,000 trajectories; the first set to form the training dataset (in-sample data) and the second one to be used later as test data in the ex-post out-of-sample analysis.

\section{B. Constructing Ambiguity Sets}

Using 5,000 training trajectories, we calculate the mean production vectors of wind farms $\mathbf{m}_{\text {srt }}$ as well as the covariance matrices of wind power forecast errors $\boldsymbol{\Sigma}_{\text {srt }}$ and $\hat{\boldsymbol{\Sigma}}_{\text {srt }}$ for each long-term scenario, representative day and hour. For example, Fig. 1 illustrates the training data in per-unit used for constructing the covariance matrix $\hat{\boldsymbol{\Sigma}}_{s r t}$ in a sample representative day under both long-term scenarios. This figure illustrates a matrix of plots in which the diagonal plots display the histograms of production forecast errors pertaining to wind farms $w 1$ and $w 2$ at hours $t$ and $t-1$. Besides, the scatter plots of corresponding forecast errors appear in the off-diagonal.

\footnotetext{
${ }^{11}$ We will explore later in Section V.F the robustness of the proposed model against the long-term uncertainty. We observe that increasing the number of long-term scenarios significantly increases the CPU time, such that the same case study with 4 long-term scenarios is solved in around 6 to 7 hours, depending on the value of confidence level. To tackle this computational issue, one may use decomposition techniques. One potential solution is to use Benders' decomposition by treating the expansion decisions as complicating (coupling) variables, e.g., in a similar way as in [14]. However, we leave it for future extensions as it is not the main focus of the current paper.
} 

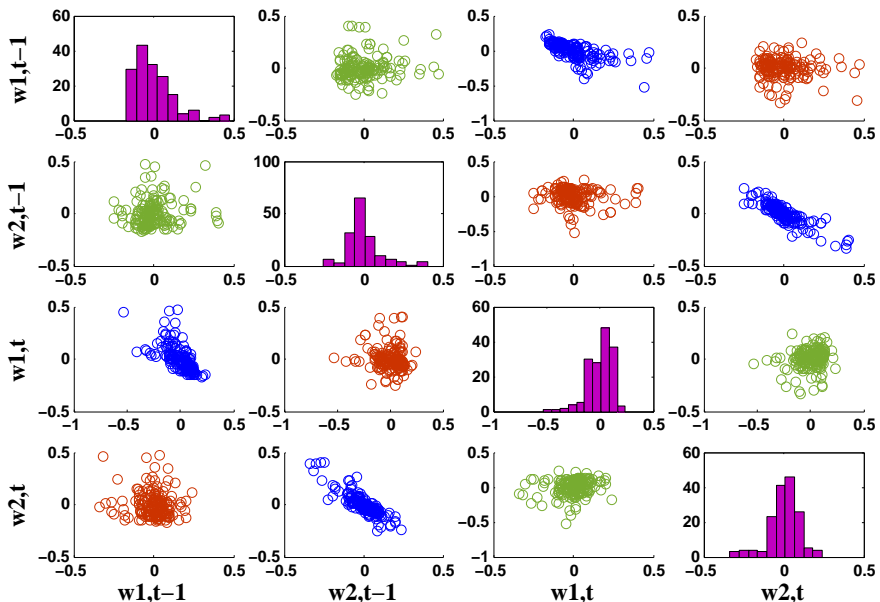

Fig. 1. Production forecast errors of wind farms $w 1$ and $w 2$ at hours $t$ and $t-$ 1 in a sample representative day. The diagonal plots ( $\mathrm{x}$-axis: in per-unit; $\mathrm{y}$-axis: frequency of occurrence) display the frequency of forecast error realizations. The off-diagonal plots (both axes in per-unit) display the realizations of two different corresponding forecast errors.

The histogram plots show the frequency distribution of each forecast error, whereas the scatter plots show the correlation of two corresponding forecast errors. The scatter plots in blue and green, respectively, show temporal and spatial correlations only. However, the scatter plots in red show the combination of temporal and spatial correlations between hours $t$ and $t-1$ and between wind farms $w 1$ and $w 2$. From the histogram plots, it is evident that the forecast error of wind production does not necessarily follow a specific probability distribution. Furthermore, it can be seen that the mean production of forecast errors is zero. Therefore, these observations match with our earlier assumptions that forecast errors have unknown distribution with a zero mean value.

\section{Benchmark Models}

To assess the performance of the proposed DRCC model, we consider a chance-constrained (CC) model as a benchmark. In this benchmark, the short-term uncertainty follows a Gaussian probability distribution with the same two moments used in the DRCC model. Therefore, there is no ambiguity set in the benchmark, and it is not necessarily robust against the short-term uncertainty if it follows any other distribution. Similar to the proposed DRCC model, the benchmark model results in a mixed-integer second-order cone problem. In fact, each chance constraint with a Gaussian distribution can be analytically reformulated as a second-order cone constraint [52], as explained in Appendix D. Note that we will consider an additional benchmark in Subsection V.G, which is a chanceconstrained optimization with no assumption on probability distribution. The chance constraints in this benchmark can no longer be analytically reformulated. Following a randomized sampling approach in [53], we will solve it as either a robust optimization or a stochastic program.

\section{In-Sample Results}

We solve the proposed DRCC model to determine the optimal generation expansion plans. For simplicity, we consider an

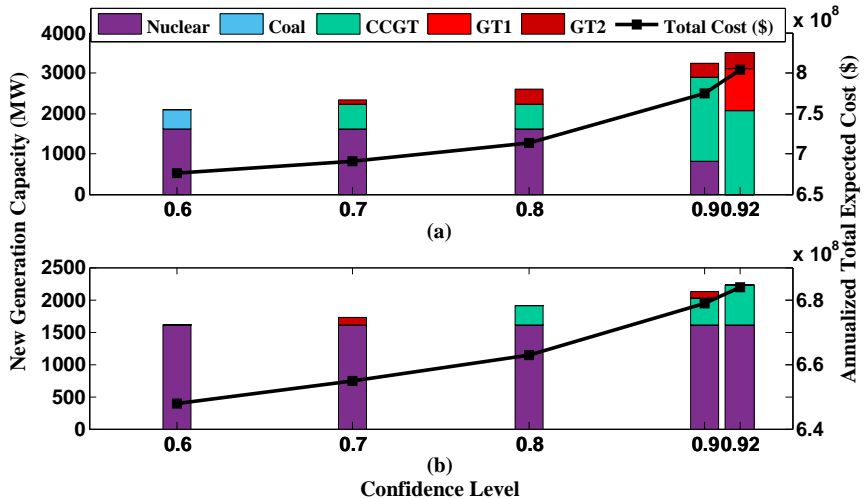

Fig. 2. In-sample results: New generation capacities to be built (left vertical axis) and annualized expected total system cost (right vertical axis) as a function of confidence level, obtained from the proposed DRCC model (upper plot) and the chance-constrained benchmark model with Gaussian probability distribution (lower plot).

identical value for $\epsilon_{i}$ and $\epsilon_{l}$ for all chance constraints, denoted as $\epsilon$, and then refer to $1-\epsilon$ as confidence level.

Fig. 2 illustrates the optimal generation capacity expansion plans (left vertical axis) and the annualized expected total system cost (right vertical axis) as a function of confidence level obtained from the proposed DRCC model (upper plot) and the benchmark CC model with Gaussian distribution (lower plot). Note that the proposed DRCC model becomes infeasible for a confidence level higher than a value between 0.95 and 0.96 , meaning that the available candidate units, even if all are built, cannot provide sufficient operational flexibility under the worst probability distribution of the short-term uncertainty, and therefore the involuntary load curtailment as an extreme recourse action will be required. In both plots, as expected, the system cost grows with increasing confidence level, as more flexible but more expensive conventional units are required to be built. For example, the proposed DRCC model suggests investing in nuclear and coal units if the confidence level is equal to 0.6 , but it drastically changes in a case with the confidence level equal to 0.92 , where this model determines flexible CCGT and GT units as the optimal generation mix to be built. In addition, a comparison of upper and lower plots in Fig. 2 points out that the proposed DRCC model is more conservative than the benchmark, resulting in a higher system cost and a higher investment in flexible generation capacity. However, we will illustrate later that the proposed model has a much better out-of-sample performance with respect to the benchmark.

In order to illustrate the effectiveness of the proposed approach in modeling the variability and uncertainty of stochastic energy sources, a sensitivity analysis with respect to the level of wind power penetration is performed. To do so, we vary the wind penetration level in both long-term scenarios from $30 \%$ to $50 \%$. The upper plot of Fig. 3 depicts the results of the proposed generation expansion model for different penetration levels. It is observed that with increasing the penetration level, a lower conventional generation capacity is required, but as expected, this new capacity is chosen among flexible technologies. This is due to the fact that a higher wind penetration implies a lower net-load, but higher 


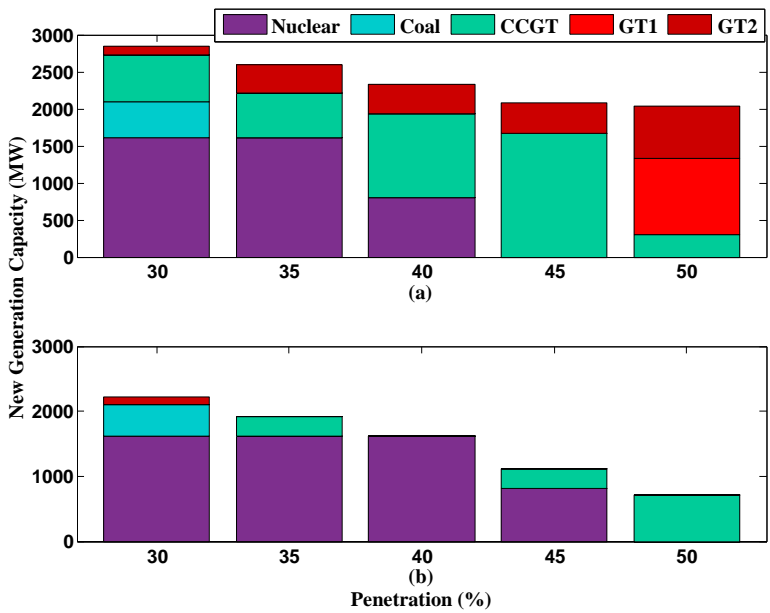

Fig. 3. In-sample results: New conventional generation capacities to be built under different values of wind power penetrations, obtained from the proposed DRCC model (upper plot) and the chance-constrained benchmark model with Gaussian probability distribution (lower plot). The confidence level is fixed to 0.8 .

variability and uncertainty in the system. As a result, the wind power penetration level not only affects the capacity of new generating units, but also changes the type of those units. The lower plot of Fig. 3 shows the results obtained from the benchmark. Compared to solutions of the DRCC model, the benchmark model fails in capturing the need for new types of flexible generating units when the wind penetration grows.

\section{E. Out-of-Sample Analysis}

Using the remaining 5,000 wind trajectories described in Subsection V.A, we perform an out-of-sample analysis in this section to evaluate the performance of the proposed DRCC model with respect to the benchmark. To this purpose, we fix the generation expansion decisions to those obtained in the insample study illustrated in Fig. 2, and then solve the tightened relaxed unit commitment problem over representative days, but using the out-of-sample wind data.

For the out-of-sample simulation, the 5,000 test trajectories are clustered using a K-means technique to achieve the same number of representative days to the one in the in-sample study, i.e., 10. However, the weighting factors of the 10 representative days in in-sample and out-of-sample studies might be different. We then randomly pick one trajectory from each cluster, i.e., representative day, such that the set of 10 selected trajectories represents the wind profile in the target year. Next, we solve the tightened relaxed unit commitment problem for the whole target year, while taking into account the extreme recourse actions, i.e., load shedding and wind spillage. We consider a value of lost load equal to $\$ 1,000 / \mathrm{MWh}$, while the wind spillage is cost free. Note that each unit commitment problem to be solved in out-ofsample simulations is a deterministic problem. We repeat this procedure until there is no trajectory left for at least one of the clusters.

For both proposed DRCC model and benchmark with Gaussian distribution, Fig. 4 shows the out-of-sample system cost in terms of mean and standard deviation as a function

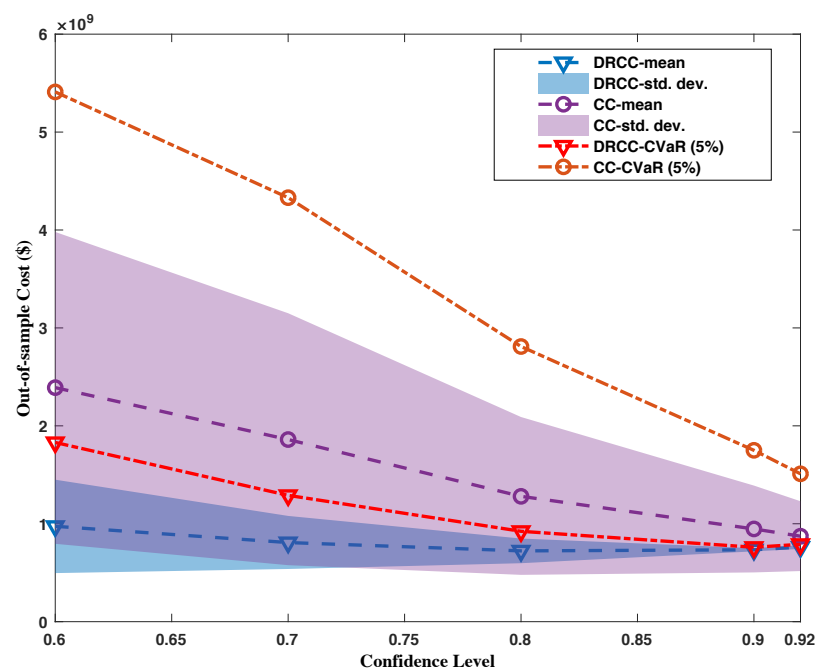

Fig. 4. Out-of-sample results: The annualized total system cost in terms of mean, standard deviation, and 5\%-CVaR obtained from the proposed DRCC model and the $\mathrm{CC}$ benchmark model with Gaussian probability distribution.

of confidence level. The range of system cost between mean plus/minus standard deviation is illustrated as a tube around the mean cost curve. In addition, this figure depicts the average system cost under the worst 5\% out-of-sample simulations, measured by a conditional value-at-risk (CVaR) metric. Let us first analyze the DRCC results. With increasing values for confidence level from 0.6 to around 0.8 , the mean cost of the DRCC model (blue dashed line) decreases, while it increases afterwards. The reason for this is that there is a relatively significant load curtailment when the confidence level is low, while it becomes zero with a higher confidence level, but with higher investment and operational costs. The trend of annual amount of load curtailment in the proposed DRCC model as a function of confidence level is given in the upper plot of Fig. 5. In addition, Fig. 4 shows that both standard deviation and 5\%-CVaR of the system cost obtained from the proposed DRCC model decrease with increasing the confidence level. Compared to this model, the CC model shows a poor out-of-sample performance, such that the mean cost, standard deviation, 5\%-CVaR, and the amount of load curtailment in that model are always higher than those in the proposed DRCC model. The mean cost of the two models in the highest confidence level in Fig. 4, i.e., 0.92, get closer, but note that such a difference will increase if a higher value for lost load is considered.

For further comparison of the two models, we present the ex-post and ex-ante violation probabilities of the solutions obtained from the DRCC model and the CC model with Gaussian distribution as given in the middle and lower plots of Fig. 5, respectively. The ex-post violation probability is achieved by solving the out-of-sample unit commitment problems: whenever there is a need for an extreme recourse action, i.e., load curtailment or wind spillage, we count it as a sample with an ex-post violation. In contrast, we do not solve any problem to compute the ex-ante violation probability: for given optimal in-sample values obtained for the tentative day-ahead schedule and participation factor of generating units, the linear 

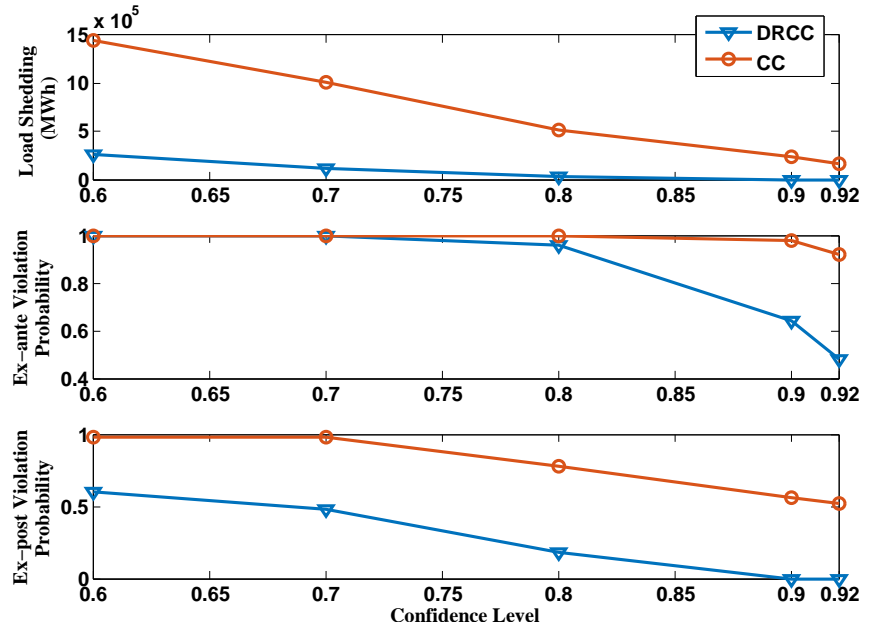

Fig. 5. Out-of-sample results: The annual amount of load curtailment (upper plot), ex-ante violation probability (middle plot), and ex-post violation probability (lower plot) obtained from the proposed DRCC model and the CC benchmark model with Gaussian probability distribution.

decision rule is applied to calculate the recourse action of each unit under each test sample. In this way, the satisfaction of chance constraints is investigated - see Appendix E for further details. According to Fig. 5, the ex-ante violation probability is higher than the ex-post one due to the fact that the linear decision rules restrict the recourse action of generating units in response to the short-term uncertainty. The key point is that with both metrics, the proposed DRCC model shows a better performance with respect to the benchmark model ${ }^{12}$.

\section{F. Additional Analyses}

In this section, we first investigate the impacts of UC constraints on planning outcomes. To do so, we compare the results achieved by the proposed model with those of a similar model including economic dispatch (ED) limits only, where all UC constraints are neglected. As expected, the ED-based model invests in more inflexible generation capacity including nuclear and coal units. As a result, the out-of-sample system cost in terms of mean and standard deviation obtained from the ED-based model is comparatively higher than that of the UCbased model. For example, in a case with a confidence level equal to 0.8 , the mean and standard deviation of the annualized system cost obtained from the out-of-sample simulation in the ED-based model are \$796 and \$241 millions, respectively. Those values in the UC-based model are \$716 and \$103 millions, respectively.

Our next analysis explores the effects of the tight relaxation of UC constraints on the out-of-sample system cost. After fixing expansion decisions to those obtained in the in-sample analysis given in Fig. 2, we run deterministic out-of-sample simulations for two cases, where the operational stage includes either exact or tightened relaxed UC constraints. For both

\footnotetext{
${ }^{12}$ It is worth mentioning that although the proposed DRCC model exhibits a good out-of-sample performance in this case study in terms of violation probability, the chance constrains in general do not restrict the severity of violations. If it is a concern, one may use the CVaR constraints rather than the chance constrains, but at the cost of additional modeling complexity and potentially a more conservative solution [54].
}

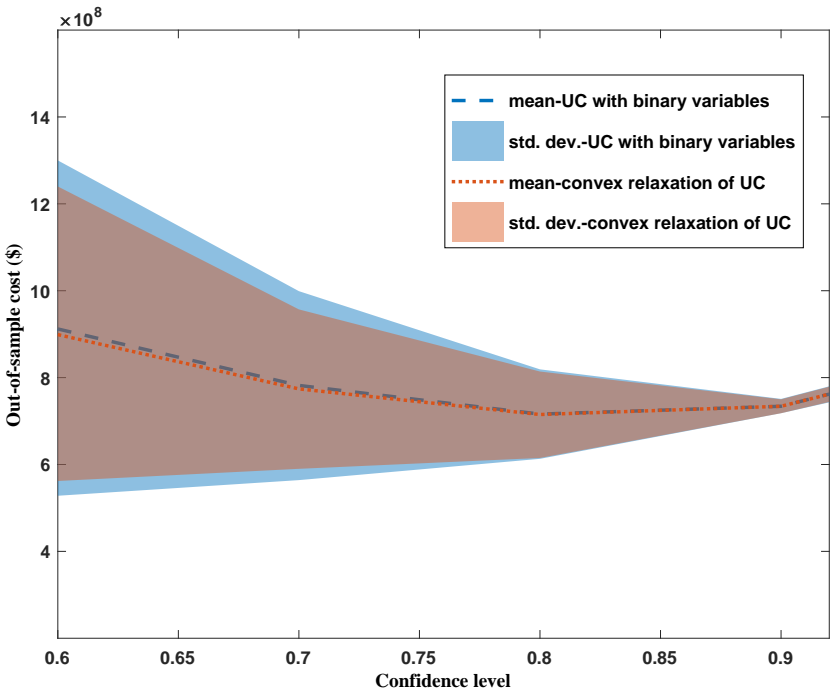

Fig. 6. Out-of-sample results: The annualized total system cost in terms of mean and standard deviation for the two cases, where the operational stage includes either exact or tightened relaxed unit commitment (UC) constraints.

cases, Fig. 6 illustrates the out-of-sample cost in terms of mean and standard deviation for different values of confidence level. This figure shows that the out-of-sample cost in case with exact UC constraints is very close to that in case with tightened relaxed UC constraints. Although this analysis does not show the impact of UC relaxation on expansion decisions, it confirms that the convex relaxation will not considerably affect the optimality of the solution obtained in the operational stage.

Another important observation is that the proposed model provides a robustness against the short-term uncertainty. However, it is not necessarily robust against the long-term uncertainty, since this uncertainty is modeled through a limited number of scenarios only. To highlight this observation, the upper plot of Fig. 7 depicts the mean and standard deviation of the out-of-sample system cost obtained from the proposed DRCC model for two different cases, namely Case I and Case II. In Case I, the two long-term scenarios for demand growth in the out-of-sample simulation are identical as those in the insample study. This is indeed a biased out-of-sample simulation in terms of long-term uncertainty. On the contrary, in Case II, we consider a forecast error in the long-term uncertainty, so that the realized load level under both scenarios are 5\% higher than that in the in-sample long-term scenarios. As observed in the upper plot of Fig. 7, this long-term forecast error results in a remarkable increase in cost in terms of both mean and standard deviation, especially for cases with relatively low confidence level. This assessment highlights the need for more advanced tools, which provide robustness not only against the short-term uncertainty, but also against the long-term one. We leave this study for future work.

As the last analysis of this subsection, we explore the robustness of the proposed model as a function of the size of the training dataset. We run our simulation with different size of training samples, varying from 3,000 to 5,000. As shown in the lower plot of Fig. 7, the proposed DRCC model is more 


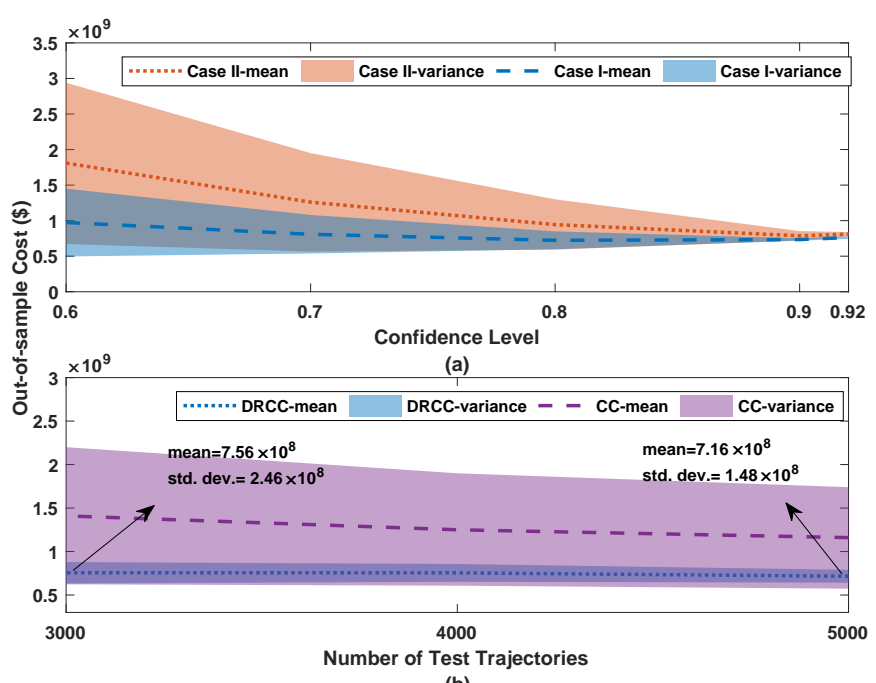

(b)

Fig. 7. Out-of-sample results: The annualized total system cost in terms of mean and standard deviation (a): obtained from the proposed DRCC model for the two cases evaluating the robustness of the proposed model against the long-term uncertainty, (b): obtained from the proposed DRCC model and the $\mathrm{CC}$ benchmark model with Gaussian probability distribution for different number of training trajectories showing the robustness of the proposed model against the size of the training dataset.

robust against the sample size compared to the benchmark $\mathrm{CC}$ model.

\section{G. Additional Benchmark: A Chance-Constrained Optimiza- tion With no Assumption on Distribution}

As mentioned earlier in Subsection V.C, we consider here an additional benchmark, which is again a chance-constrained optimization with a single distribution, but it does not follow any specific type of distribution. Note that it is not a DRCC model, as it considers a single distribution only. The distribution of this benchmark is identical to that of historical data, i.e., 5,000 in-sample trajectories allocated in 10 representative days. Unlike the benchmark in previous subsections with a Gaussian probability distribution, the chance constraints in this benchmark cannot be analytically reformulated. To solve this problem, we use a randomized sampling approach proposed in [53]. Based on this approach, depending on the inputs of the chance-constrained program, e.g., the confidence level, we randomly pick a certain number of samples from the historical data. In particular, the minimum number of samples, $M$, required is

$$
M \geqslant \frac{1}{1-\epsilon} \frac{e}{e-1}\left(1+\ln \frac{1}{\beta}\right),
$$

where $e$ is Euler number, $1-\epsilon$ is the confidence level, ensuring that the resulting uncertainty set encloses $1-\beta$ of the probability mass. It is evident that chance constraints with a higher confidence level require more samples. Similarly, the number of random samples required reduces by considering loose chance constraints. Given the set of samples picked, one can treat the problem as either a stochastic program or a robust optimization by considering a box (rectangular) uncertainty set covering all samples. The formulation of resulting stochastic program and robust optimization is provided in Appendix F.

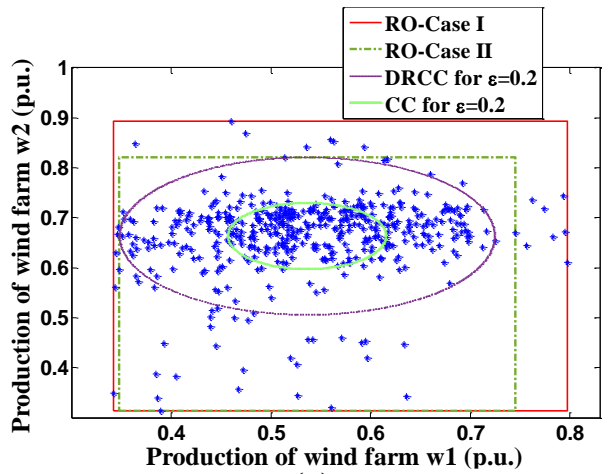

(a)

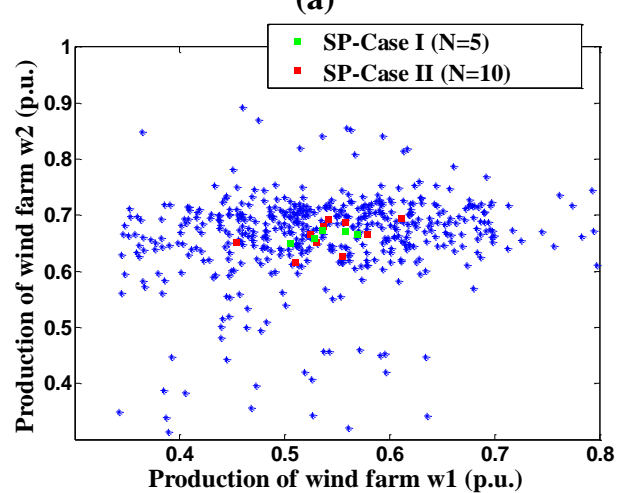

(b)

Fig. 8. In-sample scenarios (blue dots) corresponding to the power generation of two wind farms ( $w 1$ and $w 2$ ) in an arbitrarily selected hour, i.e., hour 1 in representative day 2 . Plot (a) shows two box uncertainty sets corresponding to RO-Case I and RO-Case II, as well as two ellipsoidal uncertainty sets associated with the DRCC model and the CC model with Gaussian distributions. Plot (b) illustrates the representative scenarios in SP-Case I and SP-Case II with reduced number of scenarios (green and red dots).

We take into account two cases, namely RO-Case I and RO-Case II, for the robust optimization benchmark. In ROCase I, we pick all in-sample trajectories, without using (10), aiming at obtaining the most robust solution. In RO-Case II, we consider a chance-constrained program with $1-\epsilon=0.8$ and $\beta=0.001$ for all chance constraints, and then randomly pick a number of samples according to (10). It is obvious that the number of samples picked in RO-Case II is comparatively lower than that in RO-Case I, as it corresponds to a problem with looser chance constraints. For an arbitrarily selected hour and day, i.e., hour 1 in representative day 2 , the samples picked for the two cases and their corresponding box uncertainty sets are depicted in Fig. 8(a). It is worth mentioning that there exists a close relation between chance-constrained program and robust optimization with an ellipsoidal uncertainty set, as discussed in [55] and [56]. Fig. 8(a) shows the two ellipsoidal uncertainty sets for robust optimizations, which are equivalent to the chance-constrained program with Gaussian distribution (smaller ellipsoid) and the distributionally robust chance-constrained program (larger ellipsoid). One interesting observation is that the robust optimization equivalent to DRCC (i.e., the one with larger ellipsoid) covers less samples than the robust optimization with a smaller box uncertainty set, i.e., 
RO-Case $\mathrm{II}^{13}$. Therefore, we would expect that the robust optimization corresponding to the chance-constrained benchmark model with no assumption on probability distribution provides a more conservative solution than the proposed DRCC model.

We compare the out-of-sample performance of the two robust cases RO-Case I and RO-Case II with the proposed DRCC model. The upper plot of Fig. 9 illustrates the system cost and the amount of load shedding in these three cases. It is observed that both robust cases even the one corresponding to the benchmark with a lower confidence level, i.e., RO-Case II, provide more conservative solutions than the proposed DRCC model - the system cost is higher, while the amount of load shedding is lower. This is consistent with our earlier observation in Fig. 8(a) that both box uncertainty sets cover more samples than the equivalent robust optimization to DRCC.

We now take the other potential approach with the randomly picked samples obtained from (10), and treat them as scenarios within a two-stage stochastic program, whose first stage determines the expansion decisions, and whose second stage makes the operational decisions under each scenario. Similar to the robust optimization approach, we consider two cases, namely SP-Case I and SP-Case II. By allocating the samples within 10 representative days and using a K-means clustering technique, we end up with 10 scenarios in SP-Case I and 5 scenarios in SP-Case II. Note that each scenario embodies the power generation profile of two wind farms over all hours of 10 representative days. To make a fair comparison with other approaches, we apply linear decision rules to the twostage stochastic program too, which recasts it as a single-stage model - see the corresponding formulation in Appendix F. Fig. 8(b) depicts the production of two wind farms under selected scenarios at hour 1 of representative day 2 . The lower plot of Fig. 9 illustrates the better out-of-sample performance of the proposed DRCC model compared to both stochastic cases in terms of system cost as well as amount of load shedding.

One important observation for stochastic cases is their computational requirement. The CPU time for the stochastic model with 50 and 100 scenarios is 10 and 17 hours, respectively. This is significantly higher than the solution time in the proposed DRCC model (between 2 to 3 hours depending on the value of confidence level). Our numerical analysis implies that the proposed DRCC model presents a better outof-sample solution with comparatively lower computational burden. It is obvious that by increasing the number of scenarios, the stochastic optimization will exhibit a better out-ofsample performance, but at the cost of increased computational burden. Table III provides the computational time and the number of variables and constraints in the DRCC model and the benchmarks, exhibiting the promising computational performance of the proposed DRCC model.

\section{CONCLUSION}

This paper proposes a moment-based distributionally robust chance-constrained model for generation expansion planning

\footnotetext{
${ }^{13}$ Note that this might not be the case if the randomly selected samples are enveloped by a polyhedron with more than four vertices, taking into account the potential correlation of random variables. We refer interested readers to [57] for constructing uncertainty sets for robust optimization based on available samples.
}

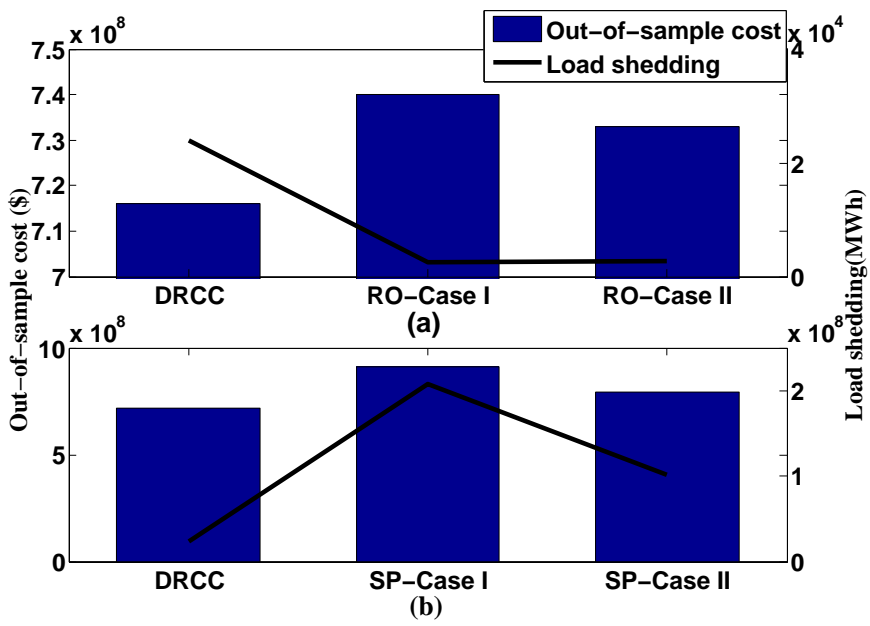

Fig. 9. Out-of-sample results: The annualized expected total system cost and the amount of load shedding obtained from the proposed DRCC model, ROCase I and RO-Case II (the upper plot), as well as SP-Case I and SP-Case II (the lower plot).

TABLE III

THE COMPUTATIONAL TIME AND THE NUMBER OF VARIABLES AND CONSTRAINTS IN DIFFERENT MODELS

\begin{tabular}{llll}
\hline \hline Model & CPU time (h) & Nr. of variables & Nr. of constraints \\
\hline DRCC & 3 & 78,744 & 518,080 \\
CC & 3 & 78,744 & 518,080 \\
RO-Case I & 8 & $1,737,624$ & $2,471,760$ \\
RO-Case II & 8 & $1,737,624$ & $2,471,760$ \\
SP-Case I & 10 & 78,744 & $1,845,680$ \\
SP-Case II & 17 & 78,744 & $3,544,540$ \\
\hline \hline
\end{tabular}

purposes. This model considers the short-term uncertainty using a family of probability distributions, and characterizes the long-term uncertainty by a set of scenarios. A tight relaxation of operational unit commitment constraints is incorporated into the model. The resulting model is a mixed-integer secondorder cone problem, which is computationally tractable. The proposed model exhibits a better out-of-sample performance compared to a chance-constrained benchmark, which considers a specific distribution of uncertainty. The future work needs to robustify the model against the long-term uncertainty. An additional uncertainty on moments can also be considered, resulting in a distributionally robust model with inexact moments. It is also of interest to explore other alternatives to construct ambiguity sets, e.g., by using a Wasserstein metric.

\section{APPENDiX A: NOMENCLATURE}

\section{A. Indices}

$i \quad$ Index for conventional generating units.

$l \quad$ Index for transmission lines.

$r \quad$ Index for representative days.

$s \quad$ Index for long-term scenarios.

$t \quad$ Index for operating hours.

\section{B. Uncertainty modeling}

$\mathcal{P}_{\text {srt }}$ Ambiguity set associated with long-term scenario $s$, representative day $r$, and hour $t$.

$\gamma_{s r t} \quad$ Random variable of forecast error at hour $t$ of representative day $r$ under long-term scenario $s$. 
$\boldsymbol{\mu}_{\text {srt }} \quad$ Mean vector of forecast error at hour $t$ of representative day $r$ under long-term scenario $s$.

$\boldsymbol{\Sigma}_{\text {srt }}$ Covariance matrix of forecast error at hour $t$ of representative day $r$ under long-term scenario $s$.

$\Psi_{\text {srt }} \quad$ Family of potential distributions associated with longterm scenario $s$, representative day $r$, and hour $t$.

$D_{\text {srt }} \quad$ Worst probability distribution of the short-term uncertainty in representative day $r$, hour $t$ under long-term scenario $s$.

\section{Parameters}

$\kappa_{r} \quad$ Weight of representative day $r$ [day].

c Production cost of generating units [\$/MWh].

$\mathbf{d}_{\text {srt }}$ Load level in the short run in representative day $r$, hour $t$ under long-term scenario $s$ [MW].

h Start-up cost of generating units [\$].

$\mathbf{H}^{[.]} \quad$ Power transfer distribution factor mapping generating units $(\mathrm{G})$, uncertain sources (W), and demand (D) on network.

$\mathbf{k} \quad$ Annualized capital cost of candidate units [\$].

$\mathbf{m}_{\text {srt }}$ Mean vector of production of uncertain sources in representative day $r$, hour $t$ under long-term scenario $s[\mathrm{MW}]$.

$\overline{\mathbf{v}}, \mathbf{v}$ Minimum up- and down-time of generating units [hour].

$\bar{f}_{l} \quad$ Capacity of transmission line $l[\mathrm{MW}]$.

$\bar{p}_{i}, \underline{p}_{i} \quad$ Maximum and minimum production level of generating unit $i[\mathrm{MW}]$.

$\bar{r}_{i}, \underline{r}_{i} \quad$ Maximum ramp-up and ramp-down capability of generating unit $i[\mathrm{MW} / \mathrm{h}]$.

$\pi_{s} \quad$ Probability of long-term scenarios $s$.

$r_{i} \quad$ Ramp rate limit of generating unit $i$ in start-up and

\section{Binary Variables} shut-down hours $[\mathrm{MW} / \mathrm{h}]$.

$\mathbf{y} \quad$ Investment decision of candidate generating units.

$u_{\text {isrt }} \quad$ Start-up status of unit $i$ in representative day $r$, hour $t$ under long-term scenario $s$.

$x_{i s r t}$ On/off commitment status of generating unit $i$ in representative day $r$, hour $t$ under long-term scenario $s$.

\section{E. Continuous Variables}

$\alpha_{i s r t} \quad$ Participation factor of unit $i$ in response to the shortterm uncertainty in representative day $r$, hour $t$ under long-term scenario $s$ [per-unit].

$p_{\text {isrt }} \quad$ Tentative day-ahead schedule of unit $i$ in representative day $r$, hour $t$ under long-term scenario $s$ [MW].

\section{APPENDIX B: REFORMULATION}

In the following three sections of this appendix, we elaborate on the reformulations of objective function (2), power balance constraint (4e), and distributionally robust chance constraints (5) and (7), respectively. Recall that we use linear decision rules in this paper, and therefore we substitute power production of each conventional generating unit $i$, i.e., $p_{i s r t}(\gamma)$ with $p_{i s r t}+\left(\mathbf{1}^{\top} \gamma\right) \alpha_{i s r t}, \forall i, s, r, t$.

\section{A. Objective Function}

By implementing linear decisions rules, the three-stage objective function (2) is written as

$$
\begin{aligned}
\min _{\mathbf{y}} \mathbf{k}^{\top} \mathbf{y} & +\sum_{s r t} \pi_{s} \max _{D \in \mathcal{P}_{s r t}} \kappa_{r} \min _{\mathbf{p}, \boldsymbol{\alpha}, \mathbf{x}, \mathbf{u}} \\
& \mathbb{E}^{D}\left[\mathbf{c}^{\top}\left(\mathbf{p}_{s r t}+\left(\mathbf{1}^{\top} \boldsymbol{\gamma}\right) \boldsymbol{\alpha}_{\text {srt }}\right)+\mathbf{h}^{\top} \mathbf{u}_{\text {srt }}\right]
\end{aligned}
$$

where $\boldsymbol{\alpha}_{s r t} \in \mathbb{R}^{G}$. Recall that the mean of the forecast error uncertainty vector $\gamma$ is assumed to be zero, i.e., $\mathbb{E}^{D}(\gamma)=\boldsymbol{\mu}=$ 0 . Therefore, the second row of (11a) reduces to $\mathbf{c}^{\top} \mathbf{p}_{\text {srt }}+$ $\mathbf{h}^{\top} \mathbf{u}_{\text {srt }}$. Now, the forecast error $\gamma$ does no longer exist in the objective function. Similarly, constraints (4e), (5) and (7) will be reformulated in the next two sections of this appendix in a way that they will not include $\gamma$. As a result, maximization operator $\max _{D \in \mathcal{P} \text { int }}$ in min-max-min objective function (11a) can be removed [29], yielding a min-min objective function, which can be combined to a single minimization function as

$$
\min _{\mathbf{y}, \mathbf{p}, \boldsymbol{\alpha}, \mathbf{x}, \mathbf{u}} \mathbf{k}^{\top} \mathbf{y}+\sum_{s, r, t} \pi_{s} \kappa_{r}\left(\mathbf{c}^{\top} \mathbf{p}_{s r t}+\mathbf{h}^{\top} \mathbf{u}_{s r t}\right) .
$$

\section{B. Power Balance Constraint}

The linear decisions rules reformulate the power balance constraint $(4 \mathrm{e})$ to

$$
\begin{aligned}
\mathbf{1}^{\top}\left(\mathbf{p}_{s r t}+\right. & \left.\left(\mathbf{1}^{\top} \gamma\right) \boldsymbol{\alpha}_{s r t}\right)+\mathbf{1}^{\top}\left(\mathbf{m}_{s r t}+\gamma\right) \\
& =\mathbf{1}^{\top} \mathbf{d}_{s r t}, \quad \forall s, r, t .
\end{aligned}
$$

In order to satisfy (12a) for all realizations of the forecast error $\gamma$, we set the first-order coefficient of $\gamma$ equal to zero [21]. Thus, by matching the zero- and first-order coefficients of $\gamma$ on both sides of (12a), we obtain

$$
\begin{aligned}
& \mathbf{1}^{\top} \boldsymbol{\alpha}_{s r t}=-\mathbf{1}, \quad \forall s, r, t, \\
& \mathbf{1}^{\top} \mathbf{p}_{s r t}+\mathbf{1}^{\top} \mathbf{m}_{s r t}=\mathbf{1}^{\top} \mathbf{d}_{s r t}, \quad \forall s, r, t .
\end{aligned}
$$

\section{Distributionally Robust Chance Constraints}

By applying Chebyshev inequality to a distributionally robust individual chance constraint as explained in [58] and [44], we can analytically reformulate distributionally robust chance constraints (5) and (7). However, one may use this reformulation with caution, as the accuracy of Chebyshev approximation might be reduced when the value of confidence level $1-\epsilon$ is very close to one. The concern is that it may obtain a unnecessarily conservative or even infeasible solution ${ }^{14}$ [29].

For example, we provide here the reformulation of distributionally robust chance constraint (5a). Other chance constraints can be analytically reformulated in the same manner. By implementing the linear decision rules, (5a) is written as

$$
\min _{D \in \mathcal{P}_{\text {srt }}} \mathbb{P}\left(p_{\text {isrt }}+\alpha_{\text {isrt }}\left(\mathbf{1}^{\top} \gamma\right) \leqslant \bar{p}_{i} x_{\text {isrt }}\right) \geqslant 1-\epsilon_{i}, \forall i, s, r, t .
$$

\footnotetext{
${ }^{14}$ To resolve this concern, an exact but more complicated reformulation is proposed in [29], which is not implemented in this paper. Reference [59] provides a comparison of the two approximate and exact reformulations by applying them to a market-clearing problem.
} 
Assuming $\boldsymbol{\mu}_{\text {srt }}=0$ while following the approach in [58] and [44] to implement Chebyshev inequality, the above chance constraint is analytically reformulated as

$p_{i s r t} \leqslant \bar{p}_{i} x_{i s r t}-\sqrt{\frac{1-\epsilon_{i}}{\epsilon_{i}}} \sqrt{\alpha_{i s r t} \mathbf{1}^{\top} \boldsymbol{\Sigma}_{s r t} \mathbf{1} \alpha_{i s r t}}, \quad \forall i, s, r, t$,

which can be eventually rewritten as

$$
p_{i s r t} \leqslant \bar{p}_{i} x_{i s r t}-\sqrt{\frac{1-\epsilon_{i}}{\epsilon_{i}}}\left\|\alpha_{i s r t} \mathbf{1}^{\top} \mathbf{\Sigma}_{\text {srt }}^{1 / 2}\right\|_{2}, \quad \forall i, s, r, t .
$$

Note that the resulting reformulation (13c) is a secondorder cone constraint. It can be reduced to a linear constraint by assuming $\alpha_{i s r t} \leqslant 0$. However, it is a strong assumption, especially in problems with network and unit commitment constraints, and we avoid taking such an assumption. Therefore, each distributionally robust chance constraint (5) and (7) is reformulated to a second-order cone constraint.

\section{ApPendix C: Resulting Model}

According to all reformulations explained in Appendix B, the resulting mixed-integer second-order cone problem for the proposed DRCC model (2)-(5) is

$$
\min _{\mathbf{y}, \mathbf{p}, \boldsymbol{\alpha}, \mathbf{x}, \mathbf{u}} \mathbf{k}^{\top} \mathbf{y}+\sum_{s, r, t} \pi_{s} \kappa_{r}\left(\mathbf{c}^{\top} \mathbf{p}_{s r t}+\mathbf{h}^{\top} \mathbf{u}_{s r t}\right)
$$

subject to:

$$
\begin{aligned}
& \text { (4a)-(4d),(4f),(6),(12b)-(12c),(13c) } \\
& p_{i s r t} \geqslant \underline{p}_{i} x_{i s r t}+\sqrt{\frac{1-\epsilon_{i}}{\epsilon_{i}}}\left\|\alpha_{i s r t} \mathbf{1}^{\top} \mathbf{\Sigma}_{s r t}^{1 / 2}\right\|_{2}, \quad \forall i, s, r, t \\
& p_{i s r t}-p_{i s r(t-1)} \leqslant \bar{r}_{i} x_{i s r(t-1)}+r_{i}\left(1-x_{i s r(t-1)}\right) \\
& -\sqrt{\frac{1-\epsilon_{i}}{\epsilon_{i}}}\left\|\mathbf{v}_{i s r t}^{\top} \hat{\boldsymbol{\Sigma}}_{s r t}^{1 / 2}\right\|_{2}, \quad \forall i, s, r, t \\
& p_{i s r t}-p_{i s r(t-1)} \geqslant-\underline{r}_{i} x_{i s r t}-r_{i}\left(1-x_{i s r t}\right) \\
& +\sqrt{\frac{1-\epsilon_{i}}{\epsilon_{i}}}\left\|\mathbf{v}_{i s r t}^{\top} \widehat{\boldsymbol{\Sigma}}_{s r t}^{1 / 2}\right\|_{2}, \quad \forall i, s, r, t \\
& \mathbf{H}_{l}^{\mathrm{G}} \mathbf{p}_{s r t}+\mathbf{H}_{l}^{\mathrm{W}} \mathbf{m}_{s r t}-\mathbf{H}_{l}^{\mathrm{D}} \mathbf{d}_{s r t} \leqslant \bar{f}_{l} \\
& -\sqrt{\frac{1-\epsilon_{l}}{\epsilon_{l}}}\left\|\left(\mathbf{H}_{l}^{\mathrm{G}} \alpha_{s r t} \mathbf{1}^{\top}+\mathbf{H}_{l}^{\mathrm{W}}\right) \mathbf{\Sigma}_{s r t}^{1 / 2}\right\|_{2}, \quad \forall l, s, r, t \\
& \mathbf{H}_{l}^{\mathrm{G}} \mathbf{p}_{s r t}+\mathbf{H}_{l}^{\mathrm{W}} \mathbf{m}_{s r t}-\mathbf{H}_{l}^{\mathrm{D}} \mathbf{d}_{s r t} \geqslant-\bar{f}_{l} \\
& \left.+\sqrt{\frac{1-\epsilon_{l}}{\epsilon_{l}}} \|\left(\mathbf{H}_{l}^{\mathrm{G}} \alpha_{s r t} \mathbf{1}^{\top}+\mathbf{H}_{l}^{\mathrm{W}}\right) \mathbf{\Sigma}_{s r t}^{1 / 2}\right) \|_{2}, \quad \forall l, s, r, t \\
& p_{i s r(t-1)} \leqslant r_{i} x_{i s r}(t-1)+\left(\bar{p}_{i}-r_{i}\right)\left(x_{i s r t}-u_{i s r t}\right) \\
& -\sqrt{\frac{1-\epsilon_{i}}{\epsilon_{i}}}\left\|\alpha_{i s r(t-1)} \mathbf{1}^{\top} \boldsymbol{\Sigma}_{s r(t-1)}^{1 / 2}\right\|_{2}, \quad \forall i, s, r, t \\
& p_{i s r t} \leqslant \bar{p}_{i} x_{i s r t}-\left(\bar{p}_{i}-r_{i}\right) u_{i s r t} \\
& -\sqrt{\frac{1-\epsilon_{i}}{\epsilon_{i}}}\left\|\alpha_{i s r t} \mathbf{1}^{\top} \boldsymbol{\Sigma}_{s r t}^{1 / 2}\right\|_{2}, \quad \forall i, s, r, t \\
& p_{i s r t}-p_{i s r(t-1)} \leqslant\left(\underline{p}_{i}+\bar{r}_{i}\right) x_{i s r t}-\left(\underline{p}_{i}+\bar{r}_{i}-r_{i}\right) u_{i s r t} \\
& -p_{i s} x_{i s r(t-1)}-\sqrt{\frac{1-\epsilon_{i}}{\epsilon_{i}}}\left\|\mathbf{v}_{i s r t}^{\top} \hat{\boldsymbol{\Sigma}}_{s r t}^{1 / 2}\right\|_{2}, \quad \forall i, s, r, t
\end{aligned}
$$

where $l s_{j} \in \mathbb{R}_{+}$and $w s_{j} \in \mathbb{R}_{+}$represent the values of load shedding and wind spillage, respectively, for sample trajectory

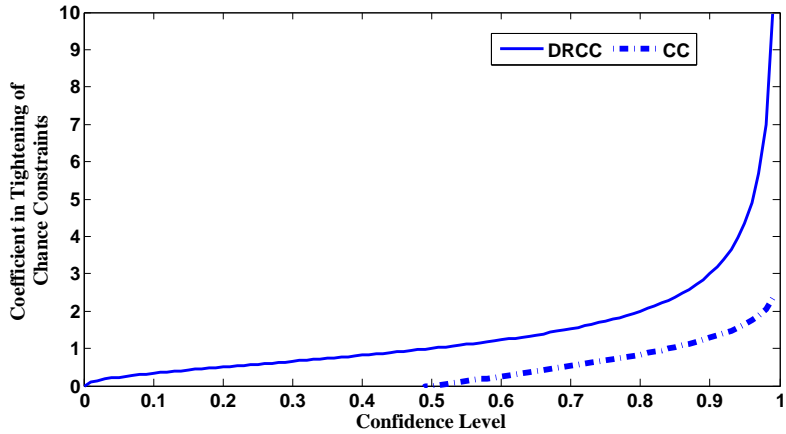

Fig. 10. The value of tightening coefficient of chance constraints as a function of confidence level $1-\epsilon$. This value in the DRCC model is $\sqrt{\frac{1-\epsilon}{\epsilon}}$, while it is equal to $\Phi^{-1}(1-\epsilon)$ in the $\mathrm{CC}$ model assuming a Gaussian probability distribution.

$$
-\left(r_{i}-\underline{r}_{i}\right) x_{i s r t}+\sqrt{\frac{1-\epsilon_{i}}{\epsilon_{i}}}\left\|\mathbf{v}_{i s r t}^{\top} \widehat{\boldsymbol{\Sigma}}_{\text {srt }}^{1 / 2}\right\|_{2}, \quad \forall i, s, r, t,
$$

where vector $\mathbf{v}_{\text {isrt }}$ is defined as

$$
\mathbf{v}_{i s r t}=\left[\begin{array}{c}
\alpha_{i s r t} \\
-\alpha_{i s r(t-1)}
\end{array}\right], \forall i, s, r, t .
$$

\section{Appendix D: Chance Constraints with Gaussian DISTRIBUTION}

Assuming a Gaussian probability distribution while assuming $\boldsymbol{\mu}_{s r t}=0, \forall s, r, t$, the arbitrarily selected chance constraint (5a) is reformulated in an exact way to a second-order cone constraint [52] as

$p_{i s r t} \leqslant \bar{p}_{i} x_{i s r t}-\Phi^{-1}\left(1-\epsilon_{i}\right) \sqrt{\alpha_{i s r t} \mathbf{1}^{\top} \boldsymbol{\Sigma}_{s r t} \mathbf{1} \alpha_{i s r t}}, \forall i, s, r, t$,

where $\Phi^{-1}(1-\epsilon)$ is the inverse cumulative distribution function. The key difference between (15) and (13b) is the coefficient in their last term. Fig. 10 illustrates the values of coefficients $\sqrt{\frac{1-\epsilon}{\epsilon}}$ and $\Phi^{-1}(1-\epsilon)$ as a function of confidence level, and thereby, their effects on tightening the chance constraints. For the same value of confidence level, it can be concluded that the DRCC model provides comparatively tighter chance constraints, which results in a more robust solution. This tightening effect is more remarkable for higher values of confidence level.

\section{APPENDix E: Violation Probabilities}

To assess ex-post and ex-ante violation probabilities, we use the test dataset containing 5,000 trajectories. The ex-post violation probability is calculated by solving the unit commitment problem a posteriori including extreme recourse actions, i.e., load shedding and wind spillage. We solve the tight relaxed unit commitment problem for each sample trajectory $j$ to calculate the violation indicator $\widehat{I}_{j}$ as

$$
\widehat{I}_{j}= \begin{cases}1 & \text { if } l s_{j}>0 \text { or } w s_{j}>0, \\ 0 & \text { otherwise, }\end{cases}
$$


$j$. We use (16) to assess the number of violations and then calculate the ex-post violation probability $\widehat{V}$ as

$$
\widehat{V}=\frac{1}{N} \sum_{j=1}^{N} \widehat{I}_{j},
$$

where $N$ is the number of sample trajectories.

Unlike the ex-post violation probability, we do not run the tight relaxed unit commitment problem to calculate the ex-ante violation probability. Given in-sample results, we calculate the recourse action of generating units for each sample trajectory by using linear decision rules. For a set of individual chance constraints in form of $\min _{D \in \mathcal{P}} \mathbb{P}\left(v^{\top} \gamma \leqslant b\right) \geqslant 1-\epsilon$, we compute the violation indicator $I_{j}$ for each trajectory $j$ as

$$
I_{j}= \begin{cases}1 & \text { if } v_{k}^{\top} \gamma_{j}>b_{k}, \\ 0 & \text { otherwise }\end{cases}
$$

where $k$ is an index for chance constraints. We investigate the satisfaction of every chance constraint by $I_{j}$. Then, we calculate the ex-ante violation probability as

$$
V=\frac{1}{N} \sum_{j=1}^{N} I_{j} .
$$

\section{APPENDIX F: RESULTING ROBUST OPTIMIZATION AND STOCHASTIC PROGRAM}

\section{A. Robust Optimization}

In order to recast the benchmark model in Section V.G as a robust optimization, we first calculate the mean value of selected samples, i.e., $\mathbf{m}_{s r t}$, for each long-term scenario $s$, representative day $r$, and hour $t$. We then construct the uncertainty set of forecast errors as $\gamma_{s r t} \in \mathcal{U}_{s r t}=\left[\gamma_{s r t}^{\min }, \gamma_{s r t}^{\max }\right]$. For simplicity, we neglect the potential correlation between random variables. Note that the mean of forecast error is zero. To formulate the robust optimization problem, the chance constraints in the proposed DRCC model should be replaced by regular constraints, meaning that for any realization within the uncertainty set $\mathcal{U}_{s r t}$, the constraints are always satisfied. For example, we provide below the formulation for the robust counterpart of (5a), imposing the capacity limit of conventional generating unit $i$. By implementing the linear decision rules, the corresponding robust constraint is written as

$$
p_{i s r t}+\alpha_{i s r t}\left(\mathbf{1}^{\top} \boldsymbol{\gamma}_{s r t}\right) \leqslant \bar{p}_{i} x_{i s r t}, \forall i, s, r, t,
$$

where $\gamma_{s r t} \in \mathcal{U}_{s r t}$. By using duality theory, the robust counterpart of (20) is formulated as

$$
\begin{aligned}
& p_{i s r t}+\boldsymbol{\eta}_{i s r t}^{\mathrm{up}^{\top}} \boldsymbol{\gamma}_{\text {srt }}^{\max }-\boldsymbol{\eta}_{i s r t}^{\mathrm{dn}^{\top}} \boldsymbol{\gamma}_{s r t}^{\min } \leqslant \bar{p}_{i} x_{i s r t}, \forall i, s, r, t \\
& \eta_{z i s r t}^{\text {up }}-\eta_{z i s r t}^{\mathrm{dn}}=\alpha_{i s r t}, \quad \forall z, i, s, r, t \\
& \eta_{z i s r t}^{\text {up }} \geqslant 0, \quad \eta_{z i s r t}^{\mathrm{dn}} \geqslant 0, \quad \forall z, i, s, r, t .
\end{aligned}
$$

where $\boldsymbol{\eta}_{i s r t}^{\text {up }} \in \mathbb{R}^{Z}$ and $\boldsymbol{\eta}_{\text {isrt }}^{\mathrm{dn}} \in \mathbb{R}^{Z}$ are dual variables, and $z$ is an index for wind farms. A further elaboration on the derivation of linear robust counterpart is given in [60]-[62]. Given the fact that the mean of forecast error is zero, the objective function of the resulting robust optimization reduces to the one of the proposed DRCC model, i.e., (11b).

\section{B. Stochastic Programming}

By clustering samples in Subsection V.G, we obtain a set of short-term scenarios $\omega \in \Omega_{\text {srt }}$ associated with long-term scenario $s$, representative day $r$, and hour $t$. According to the number of samples in each cluster, a probability $v_{s r t \omega} \in \mathbb{R}_{+}$is assigned to each short-term scenario $\omega$ such that $\sum_{\omega} v_{\text {srt } \omega}=$ $1, \forall s, r, t$. To make a fair comparison among models, we implement linear decisions rules in the stochastic benchmark. Under each short-term scenario, all operational constraints need to be satisfied. For example, the capacity constraint of unit $i$ imposed by (5a) in the proposed DRCC model is written as

$$
p_{i s r t}+\alpha_{i s r t}\left(\mathbf{1}^{\top} \gamma_{s r t \omega}\right) \leqslant \bar{p}_{i} x_{i s r t}, \quad \forall i, s, r, t, \forall \omega \in \Omega_{s r t}
$$

where $\gamma_{s r t \omega} \in \mathbb{R}^{Z}$ is the vector of forecast errors under shortterm scenario $\omega$. Finally, the objective function of the resulting stochastic program is

$$
\begin{aligned}
& \min _{\mathbf{y}, \mathbf{p}, \boldsymbol{\alpha}, \mathbf{x}, \mathbf{u}} \mathbf{k}^{\top} \mathbf{y}+ \\
& \sum_{s r t \omega} \pi_{s} \kappa_{r}\left[\mathbf{c}^{\top}\left(\mathbf{p}_{s r t}+v_{s r t w}\left(\mathbf{1}^{\top} \boldsymbol{\gamma}_{s r t w}\right) \boldsymbol{\alpha}_{s r t}\right)+\mathbf{h}^{\top} \mathbf{u}_{s r t}\right] .
\end{aligned}
$$

\section{ACKNOWLEDGMENT}

The authors would like to thank the three anonymous reviewers. We also thank S.-E. Fleten (Norwegian University of Science and Technology) and Y. Dvorkin (New York University) for their valuable suggestions and comments, and participants at the Mathematics of Energy Systems Workshop at Isaac Newton Institute, University of Cambridge, March 2019, for helpful discussions.

\section{REFERENCES}

[1] A. J. Conejo, L. Baringo, S. J. Kazempour, and A. S. Siddiqui, Investment in Electricity Generation and Transmission: Decision Making Under Uncertainty. Springer, Switzerland, 2016.

[2] J. Mays, D. P. Morton, and R. P. O'Neill, "Asymmetric risk and fuel neutrality in electricity capacity markets," Nature Energy, Oct. 2019.

[3] X. Zhang and A. J. Conejo, "Robust transmission expansion planning representing long- and short-term uncertainty," IEEE Trans. Power Syst., vol. 33, no. 2, pp. 1329-1338, Mar. 2018.

[4] S. Jin, S. M. Ryan, J. Watson, and D. L. Woodruff, "Modeling and solving a large-scale generation expansion planning problem under uncertainty," Energy Systems, vol. 2, no. 3-4, pp. 209-242, Nov. 2011.

[5] S. Jin, A. Botterud, and S. M. Ryan, "Temporal versus stochastic granularity in thermal generation capacity planning with wind power," IEEE Trans. Power Syst., vol. 29, no. 5, pp. 2033-2041, Sep. 2014.

[6] S. Dehghan, N. Amjady, and A. Kazemi, "Two-stage robust generation expansion planning: A mixed integer linear programming model," IEEE Trans. Power Syst., vol. 29, no. 2, pp. 584-597, Mar. 2014.

[7] F. Maggioni, M. Cagnolari, and L. Bertazzi, "The value of the right distribution in stochastic programming with application to a newsvendor problem," Computat. Manage. Sci., pp. 1-20, 2019, to be published.

[8] W. Wiesemann, D. Kuhn, and M. Sim, "Distributionally robust convex optimization," Oper. Res., vol. 62, no. 6, pp. 1358-1376, Nov.-Dec. 2014.

[9] T. Zhang, R. Baldick, and T. Deetjen, "Optimized generation capacity expansion using a further improved screening curve method," Elect. Power Syst. Res., vol. 124, pp. 47-54, Jul. 2015.

[10] B. S. Palmintier and M. D. Webster, "Impact of operational flexibility on electricity generation planning with renewable and carbon targets," IEEE Trans. Sustain. Energy, vol. 7, no. 2, pp. 672-684, Apr. 2016.

[11] J. Ma, V. Silva, R. Belhomme, D. S. Kirschen, and L. F. Ochoa, "Evaluating and planning flexibility in sustainable power systems," IEEE Trans. Sustain. Energy, vol. 4, no. 1, pp. 200-209, Jan. 2013. 
[12] B. Hua, R. Baldick, and J. Wang, "Representing operational flexibility in generation expansion planning through convex relaxation of unit commitment," IEEE Trans. Power Syst., vol. 33, no. 2, pp. 2272-2281, Mar. 2018.

[13] A. Shortt, J. Kiviluoma, and M. O'Malley, "Accommodating variability in generation planning," IEEE Trans. Power Syst., vol. 28, no. 1, pp. 158-169, Feb. 2013.

[14] A. Schwele, J. Kazempour, and P. Pinson, "Do unit commitment constraints affect generation expansion planning? A scalable stochastic model," Energy Systems, Jan. 2019, to be published.

[15] E. Delage and Y. Ye, "Distributionally robust optimization under moment uncertainty with application to data-driven problems," Oper. Res., vol. 58, no. 3, pp. 595-612, May-Jun. 2010.

[16] G. A. Hanasusanto, V. Roitch, D. Kuhn, and W. Wiesemann, "Ambiguous joint chance constraints under mean and dispersion information," Oper. Res., vol. 65, no. 3, pp. 751-767, May-Jun. 2017.

[17] S. Zymler, D. Kuhn, and B. Rustem, "Distributionally robust joint chance constraints with second-order moment information," Math. Program., vol. 137 , no. $1-2$, pp. 167-198, Nov. 2011.

[18] G. A. Hanasusanto and D. Kuhn, "Conic programming reformulations of two-stage distributionally robust linear programs over Wasserstein balls," Oper. Res., vol. 66, no. 3, pp. 849-869, May-Jun. 2018.

[19] P. Mohajerin Esfahani and D. Kuhn, "Data-driven distributionally robust optimization using the Wasserstein metric: Performance guarantees and tractable reformulations," Math. Program., Jul. 2017.

[20] H. Rahimian and S. Mehrotra, "Distributionally robust optimization: A review," 2019. [Online]. Available: https://arxiv.org/abs/1908.05659

[21] D. Kuhn, W. Wiesemann, and A. Georghiou, "Primal and dual linear decision rules in stochastic and robust optimization," Math. Program., vol. 130, no. 1, pp. 177-209, Nov. 2011.

[22] S. Pineda, J. M. Morales, and T. K. Boomsma, "Impact of forecast errors on expansion planning of power systems with a renewables target," Eur. J. Oper. Res., vol. 248, no. 3, pp. 1113-1122, Feb. 2016.

[23] D. Alvarado, A. Moreira, R. Moreno, and G. Strbac, "Transmission network investment with distributed energy resources and distributionally robust security," IEEE Trans. Power Syst., vol. 34, no. 6, pp. 5157-5168, Nov. 2019.

[24] D. Pozo, A. Street, and A. Velloso, "An ambiguity-averse model for planning the transmission grid under uncertainty on renewable distributed generation," in Power Systems Computation Conf., Jun. 2018, pp. 1-7.

[25] C. Zhao and R. Jiang, "Distributionally robust contingency-constrained unit commitment," IEEE Trans. Power Syst., vol. 33, no. 1, pp. 94-102, Jan. 2018

[26] P. Xiong, P. Jirutitijaroen, and C. Singh, "A distributionally robust optimization model for unit commitment considering uncertain wind power generation," IEEE Trans. Power Syst., vol. 32, no. 1, pp. 39-49, Jan. 2017.

[27] Y. Chen et al., "A distributionally robust optimization model for unit commitment based on Kullback-Leibler divergence," IEEE Trans. Power Syst., vol. 33, no. 5, pp. 5147-5160, Sep. 2017.

[28] W. Wei, F. Liu, and S. Mei, "Distributionally robust co-optimization of energy and reserve dispatch," IEEE Trans. Sustain. Energy, vol. 7, no. 1, pp. 289-300, Jan. 2016

[29] W. Xie and S. Ahmed, "Distributionally robust chance constrained optimal power flow with renewables: A conic reformulation," IEEE Trans. Power Syst., vol. 33, no. 2, pp. 1860-1867, Mar. 2018.

[30] R. Mieth and Y. Dvorkin, "Data-driven distributionally robust optimal power flow for distribution systems," IEEE Control Systems Letters, vol. 2, no. 3, pp. 363-368, Jul. 2018.

[31] Y. Guo, K. Baker, E. Dall'Anese, Z. Hu, and T. H. Summers, "Databased distributionally robust stochastic optimal power flow-Part I: Methodologies," IEEE Trans. Power Syst., vol. 34, no. 2, pp. 14831492, Mar. 2019.

[32] C. Ordoudis, V. A. Nguyen, D. Kuhn, and P. Pinson, "Energy and reserves dispatch with distributionally robust joint chance constraints," 2018. [Online]. Available: http://www.optimizationonline.org/DB_HTML/2018/12/6962.html

[33] X. Han, E. G. Kardakos, and G. Hug, "A distributionally robust bidding strategy for a wind power plant," Elect. Power Syst. Res., vol. 177, p. 105986, Dec. 2019.

[34] A. B. Philpott, V. L. de Matos, and L. Kapelevich, "Distributionally robust SDDP," Computat. Manage. Sci., vol. 15, no. 3-4, pp. 431-454, Oct. 2018.

[35] F. Qiu and J. Wang, "Distributionally robust congestion management with dynamic line ratings," IEEE Trans. Power Syst., vol. 30, no. 4, pp. 2198-2199, Jul. 2015.
[36] Z. Shi, H. Liang, S. Huang, and V. Dinavahi, "Distributionally robust chance-constrained energy management for islanded microgrids," IEEE Trans. Smart Grid, vol. 10, no. 2, pp. 2234-2244, Mar. 2019.

[37] A. Zare, C. Y. Chung, J. Zhan, and S. O. Faried, "A distributionally robust chance-constrained MILP model for multistage distribution system planning with uncertain renewables and loads," IEEE Trans. Power Syst., vol. 33, no. 5, pp. 5248-5262, Sep. 2018.

[38] F. H. Murphy and Y. Smeers, "Generation capacity expansion in imperfectly competitive restructured electricity markets," Oper. Res., vol. 53 , no. 4, pp. 646-661, 2005.

[39] P. Maghouli, S. H. Hosseini, M. Oloomi Buygi, and M. Shahidehpour, "A scenario-based multi-objective model for multi-stage transmission expansion planning," IEEE Trans. Power Syst., vol. 26, no. 1, pp. 470478, Feb. 2011

[40] S. J. Kazempour, A. J. Conejo, and C. Ruiz, "Strategic generation investment using a complementarity approach," IEEE Trans. Power Syst., vol. 26, no. 2, pp. 940-948, May 2011.

[41] K. Poncelet, H. Höschle, E. Delarue, A. Virag, and W. D'haeseleer, "Selecting representative days for capturing the implications of integrating intermittent renewables in generation expansion planning problems," IEEE Trans. Power Syst., vol. 32, no. 3, pp. 1936-1948, May 2017.

[42] Y. Liu, R. Sioshansi, and A. J. Conejo, "Hierarchical clustering to find representative operating periods for capacity-expansion modeling," IEEE Trans. Power Syst., vol. 33, no. 3, pp. 3029-3039, May 2018.

[43] D. A. Tejada-Arango, M. Domeshek, S. Wogrin, and E. Centeno, "Enhanced representative days and system states modeling for energy storage investment analysis," IEEE Trans. Power Syst., vol. 33, no. 6, pp. 6534-6544, Nov. 2018

[44] Y. Zhang, S. Shen, and J. L. Mathieu, "Distributionally robust chanceconstrained optimal power flow with uncertain renewables and uncertain reserves provided by loads," IEEE Trans. Power Syst., vol. 32, no. 2 , pp. 1378-1388, Mar. 2017.

[45] B. Li, R. Jiang, and J. L. Mathieu, "Distributionally robust chance constrained optimal power flow assuming unimodal distributions with misspecified modes," IEEE Trans. Control Netw. Syst., vol. 6, no. 3, pp. 1223-1234, Sep. 2019.

[46] B. Hua and R. Baldick, "A convex primal formulation for convex hull pricing," IEEE Trans. Power Syst., vol. 32, no. 5, pp. 3814-3823, Sep. 2017.

[47] H.-P. Chao, "Incentives for efficient pricing mechanism in markets with non-convexities," J. Reg. Econ., vol. 56, no. 1, pp. 33-58, Aug. 2019.

[48] K. Pan and Y. Guan, "A polyhedral study of the integrated minimumup/-down time and ramping polytope," arXiv preprint arXiv:1604.02184, 2016.

[49] A. Georghiou, W. Wiesemann, and D. Kuhn, "Generalized decision rule approximations for stochastic programming via liftings," Math. Program., vol. 152, no. 1, pp. 301-338, Aug. 2015.

[50] I. Peña, C. B. Martinez-Anido, and B. Hodge, "An extended IEEE 118bus test system with high renewable penetration," IEEE Trans. Power Syst., vol. 33, no. 1, pp. 281-289, Jan. 2018.

[51] [Online]. Available: https://github.com/farzanehpourahmadi/DRCC.git

[52] D. Bienstock, M. Chertkov, and S. Harnett, "Chance-constrained optimal power flow: Risk-aware network control under uncertainty," SIAM Review, vol. 56, no. 3, pp. 461-495, 2014.

[53] K. Margellos, P. Goulart, and J. Lygeros, "On the road between robust optimization and the scenario approach for chance constrained optimization problems," IEEE Trans. Autom. Control, vol. 59, no. 8, pp. 2258-2263, Aug. 2014.

[54] B. P. G. Van Parys, D. Kuhn, P. J. Goulart, and M. Morari, "Distributionally robust control of constrained stochastic systems," IEEE Trans. Autom. Control, vol. 61, no. 2, pp. 430-442, Feb. 2016.

[55] G. C. Calafiore and L. El Ghaoui, "On distributionally robust chanceconstrained linear programs," J. Optim. Theory Appl., vol. 130, no. 1, pp. 1-22, Jul 2006.

[56] B. L. Gorissen, İ. Yanıkoğlu, and D. den Hertog, "A practical guide to robust optimization," Omega, vol. 53, pp. 124-137, Jun. 2015.

[57] D. Bertsimas, V. Gupta, and N. Kallus, "Data-driven robust optimization," Math. Program., vol. 167, no. 2, pp. 235-292, Feb. 2018.

[58] M. Wagner, "Stochastic 0-1 linear programming under limited distributional information," Oper. Res. Lett., vol. 36, no. 2, pp. 150-156, Mar. 2008.

[59] Y. Dvorkin, "A chance-constrained stochastic electricity market," 2019. [Online]. Available: https://arxiv.org/abs/1906.06963

[60] D. Bertsimas and M. Sim, "Tractable approximations to robust conic optimization problems," Math. Program., vol. 107, no. 1-2, pp. 5-36, Jun. 2006. 
[61] F. Pourahmadi, H. Heidarabadi, S. H. Hosseini, and P. Dehghanian, "Dynamic uncertainty set characterization for bulk power grid flexibility assessment," IEEE Systems Journal, pp. 1-11, 2019.

[62] R. A. Jabr, "Adjustable robust OPF with renewable energy sources," IEEE Trans. Power Syst., vol. 28, no. 4, pp. 4742-4751, Nov. 2013. 